\title{
Numerical investigation of large-scale vortices in an array of cylinders in axial flow
}

\author{
Laurent De Moerloose ${ }^{\mathrm{a}, *}$, Pieter Aerts ${ }^{\mathrm{a}}$, Jeroen De Ridder ${ }^{\mathrm{a}}$, Jan \\ Vierendeels $^{\mathrm{a}, \mathrm{b}}$, Joris Degroote ${ }^{\mathrm{a}, \mathrm{b}}$ \\ ${ }^{a}$ Department of Flow, Heat and Combustion Mechanics, Faculty of Engineering and \\ Architecture, Ghent University, Sint-Pietersnieuwstraat 41-B4, 9000 Ghent, Belgium \\ ${ }^{b}$ Flanders Make, Belgium
}

\begin{abstract}
Axial flow around an array of cylinders is commonly encountered in nuclear reactors and heat exchangers. This geometry is subject to important flow instabilities. The chaotic flow fluctuations due to turbulence are not the only source of vortex structures: large-scale vortices have also been observed, both experimentally and numerically. The periodic pressure fluctuations caused by the coherent vortex structures are possibly a source of fretting and fatigue in the aforementioned applications. In order to comprehend this phenomenon, LargeEddy Simulations are performed on a numerical domain containing a single rigid cylinder with periodic boundary conditions, representative for a cylinder in an infinite square array. The research in this paper mainly focuses on the influence of the cylinder spacing, which is analyzed by calculating the Cross Spectral Density (CSD) function of the cylinder wall pressure for different cylinder spacings. The spectral analysis shows that the amplitude of the pressure fluctuations increases up to a well-determined intercylinder gap, after which it decreases exponentially for incrementing gap size. With the weakening of the instability, the location on the cylinder circumference where the maximum pressure amplitude occurs, changes as well. Finally, it is shown that the coherent vortices are transported as a whole at a convection speed which is dependent on the cylinder spacing. An updated model for this convection speed is proposed.
\end{abstract}

Keywords: Flow-induced vibrations, Kelvin-Helmholtz instability, Large-Eddy Simulations, spectral analysis

\section{Introduction}

An array of cylinders subjected to axial flow is encountered in numerous applications. Of special interest are heat exchanger geometries in general (Kakaç

\footnotetext{
*Corresponding author. Tel. +32 926432 97, Fax. +3292643590

Email address: laurent.demoerloose@ugent.be (Laurent De Moerloose)
} 
et al. (2012)) and nuclear reactor core arrays specifically (Païdoussis (1982)). The problem of fluid-elastic instabilities in both cross-flow (Pettigrew and Taylor (2003)) and axial flow (Au-Yang (2001); Païdoussis (1998)) has been investigated analytically, experimentally and numerically. Most analyses focus on the buckling behaviour of a single cylinder (Modarres-Sadeghi et al. (2007)) or of an array of cylinders. Chaotic dynamics, due to turbulence-induced vibrations, are also analyzed extensively (Curling and Païdoussis (1992); Païdoussis and Botez (1993)). However, the tube bundle geometry gives rise to non-chaotic flow phenomena as well. The earliest recollection of the existence of coherent vortices dates back to Nikuradse (1930). The secondary flow described in that report finds its origin in nonuniform turbulent mixing in a non-circular duct, but is relatively weak compared to the vortex street which dominates the velocity field. A schematic representation is shown in Figure 1, which indicates that the flow instability is related to a cross-flow mechanism across the gap between two adjacent cylinders, creating coherent vortices on either side of the gap. The vortex street is not stationary, but is transported through the flow. The symbol $U_{c}$ denotes the convection speed of the coherent structures. An example of the transverse velocity component across a cylinder gap region - obtained through the Large-Eddy Simulations described in this paper - is shown in Figure 1. Both the axial flow speed difference and the cross-flow fluctuations cause pressure oscillations on the cylinder wall. As a result, the cylinder wall pressure distribution is greatly dependent on the large-scale vortices. This provides the opportunity to analyze the vortex street by considering the cylinder wall pressure, which is the subject of this paper. 


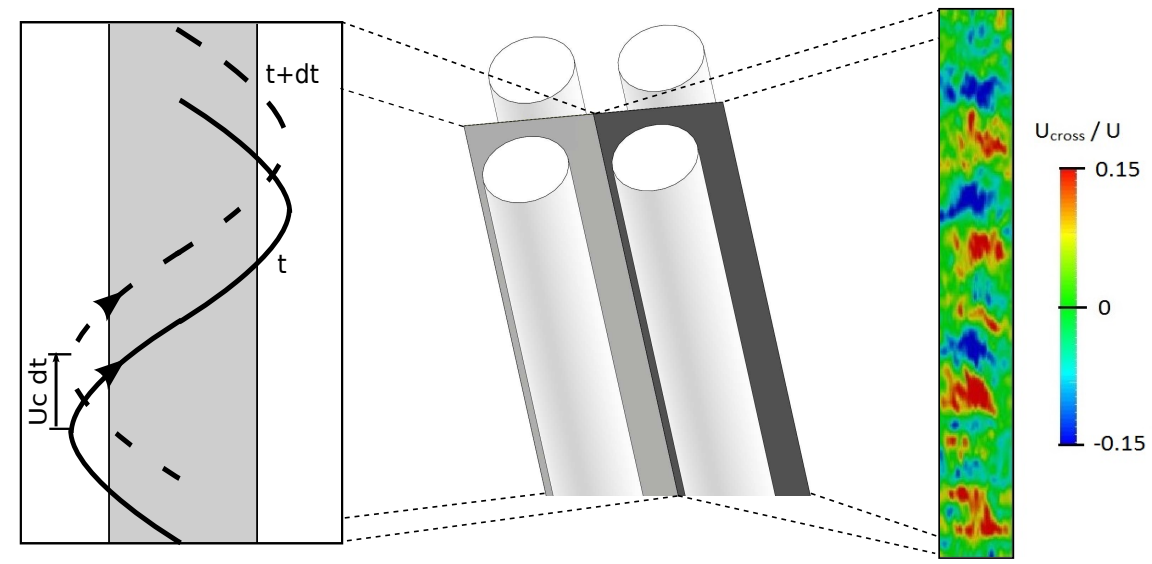

Figure 1: (Left) Schematic representation of the cross-flow mechanism in a plane located between two cylinders. The grey area represents the cylinder wall located behind the depicted flow region. The thick black line is a flow path due to the cross-flow across the gap region, neglecting turbulent fluctuations. The dotted line shows the same flow path an instant $d t$ later, assuming the large-scale vortices are transported with an axial convection speed $U_{c}$. (Center) Three-dimensional representation of the square cylinder array. (Right) An example of the cross-flow velocity component in a plane located in between two cylinders. Results are obtained with a Large-Eddy Simulation.

The large-scale vortices are related to the close spacing of the cylinders. Consider the schematic top view of a square cylinder array in Figure 2. The drawn array is assumed to be infinitely wide and long, i.e. boundary and entrance effects are not taken into account. Two flow regions are distinguished: the gap region $(\mathrm{g})$, located in between two adjacent cylinders, and the subchannel region (s), positioned in the middle of four cylinders. Due to the smaller flow area, the influence of the cylinder wall friction on the flow is stronger in the gap region than in the subchannel region. Consequently, the axial flow velocity in the subchannel region is higher than in the gap region. The flow velocity difference between a gap region and a neighbouring subchannel region leads to the existence of a Kelvin-Helmholtz instability. 


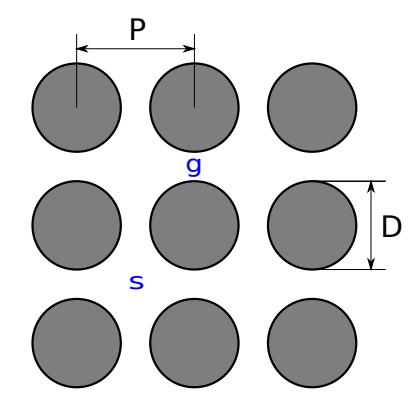

Figure 2: Schematic view of the square array of cylinders (top view). The blue letters indicate flow regions: gap (g) and subchannel (s). The black capital letters represent the geometrial parameters: diameter $(\mathrm{D})$ and pitch $(\mathrm{P})$.

A substantial number of articles describe the flow field in an array of cylinders subjected to axial flow. At first, experimental measurements have provided some insight in the flow dynamics. Curling and Païdoussis (1992) developed a correlation for the frequency spectrum of the turbulent wall-pressure field in a square array of cylinders. This empirical correlation is based on measurements of water flowing through an apparatus containing four cylinders and eight halfcylinders and was consequently only verified for a single pitch-over-diameterratio $(P / D)$. Möller (1991) analyzed the air flow through a rectangular channel containing four tubes and concluded that the frequency at which the pressure amplitude becomes maximal, is solely dependent on the geometry of the flow. He proposed a correlation to quantify this hypothesis, which will be discussed later. Finally, Guellouz and Tavoularis (2000) performed windtunnel tests on a rectangular channel containing a single cylinder and measured the convection speed $U_{c}$ of the occurring flow instability in the gap region by smoke injection. As a result, they obtained a correlation for the convection speed as a function of the $P / D$-ratio, which will be discussed in Section 5 .

With the improved computational power, more recent sources in literature apply Computational Fluid Dynamics (CFD) to investigate the large-scale vortices in an array of cylinders. Several authors apply an Unsteady ReynoldsAveraged Navier-Stokes (URANS) methodology. Merzari et al. (2008) performed URANS calculations with anisotropic turbulence modelling on a triangular cylinder array and concluded that URANS accurately predicts the amplitude and temporal frequency of the large-scale vortices, but not the spatial wavelength. This conclusion is based on the comparison of numerical and experimental results on a $P / D$-ratio of 1.06. In contrast, Yan and $\mathrm{Yu}$ (2011) calculated the flow through a similar geometry with Reynolds stress modelling and for different $P / D$-ratios and came to the conclusion that the amplitude of the large-scale vortices becomes maximal at a $P / D$-ratio equal to 1.03 .

Despite the large amount of research, few investigations were performed on a square array of cylinders subjected to axial flow. The experimental investi- 
gations by Möller (1991) and Guellouz and Tavoularis (2000) do not consider a two-dimensional array of cylinders. On the other hand, the numerical investigations by Merzari et al. (2008) and Yan and Yu (2011) consider a triangular array. Although this geometry is commonly encountered in Boiling Water Reactors (Païdoussis (1998)) and new nuclear reactor prototypes such as MYRRHA (Merzari et al. (2016)), most nuclear reactors worldwide are Pressurized Water Reactors and thus contain a square array, which is therefore equally important to analyze. Additionally, it should be noted that LES methods are generally more accurate than URANS (Piomelli (2012)), but only a few publications report LES results. An example is the work by Abbasian et al. (2010), who used a Smagorinsky model to compute the flow in a finite triangular array for a $P / D$ ratio equal to 1.08 , without investigating the influence of the $P / D$-ratio on the flow instability.

In this paper, the flow instability in a square array geometry is investigated with LES. Spectral analysis of the pressure profile on the cylinder wall reveals key aspects of the Kelvin-Helmholtz instability. The aim of this investigation is to provide a thorough understanding of these characteristics and, more specifically, how the cylinder spacing affects the flow field in a square array of cylinders.

This paper is organized as follows. Section 2 introduces the mesh characteristics as well as the applied numerical methods. Section 3 is devoted to the grid convergence analysis and the investigation of the influence of numerical parameters on the flow field. In Sections 4 and 5, the results are presented and discussed. Finally, the conclusions are given in Section 6. 


\begin{tabular}{|c|c|c|c|}
\hline \multicolumn{4}{|c|}{ Nomenclature } \\
\hline CFD & Computational Fluid Dynamics & LES & Large-Eddy Simulation \\
\hline CSD & Cross-Spectral Density & PSD & Power Spectral Density \\
\hline DFT & Discrete Fourier Transform & URANS & Unsteady Reynolds-Averaged Navier-Stokes \\
\hline$A_{\text {flow }}$ & through-flow area $\left[\mathrm{m}^{2}\right]$ & $S_{i j}$ & shear stress tensor $[1 / s]$ \\
\hline$A_{\text {wall }}$ & wall surface area $\left[\mathrm{m}^{2}\right]$ & $S t$ & Strouhal number [-] \\
\hline$C_{S}$ & Smagorinsky model parameter [-] & $t$ & time $[s]$ \\
\hline$f_{L M}, f_{M M}$ & Smagorinsky model quantities & $T$ & number of considered time steps [-] \\
\hline & cylinder diameter $[m]$ & $u$ & instantaneous velocity $[\mathrm{m} / \mathrm{s}]$ \\
\hline$D_{h}$ & hydraulic diameter $[m]$ & $U$ & mean velocity $[\mathrm{m} / \mathrm{s}]$ \\
\hline$f_{\min }$ & dimensional frequency $[\mathrm{Hz}]$ & & convection velocity $[\mathrm{m} / \mathrm{s}]$ \\
\hline$G$ & tophat filtering function & $(x, y, z)$ & cartesian coordinate system $\left[\left(m,{ }^{\circ}, m\right)\right]$ \\
\hline$h$ & cell width $[m]$ & $x_{i}$ & general spatial coordinate $[\mathrm{m}]$ \\
\hline$k$ & discrete frequency variable [-] & $\gamma$ & coherence function [-] \\
\hline$L$ & domain length $[m]$ & $\Delta$ & LES filter width $[m]$ \\
\hline$n$ & discrete time variable $[-]$ & $\mu$ & dynamic viscosity $[P a . s]$ \\
\hline$N$ & number of divisions [-] & $\nu$ & kinematic viscosity $\left[\mathrm{m}^{2} / \mathrm{s}\right]$ \\
\hline$p$ & (wall) pressure $[\mathrm{Pa}]^{\mathrm{l}}$ & $\nu_{t}$ & turbulent kinematic viscosity $\left[\mathrm{m}^{2} / \mathrm{s}\right]$ \\
\hline$P$ & cylinder pitch $[m]$ & $\rho$ & fluid density $\left[\mathrm{kg} / \mathrm{m}^{3}\right]$ \\
\hline$P(\theta, z, S t)$ & Fourier variable of the pressure $[\mathrm{Pa}]$ & $\tau_{i j}$ & residual stress tensor $\left[\mathrm{m}^{2} / \mathrm{s}^{2}\right]$ \\
\hline $\begin{array}{l}\operatorname{Re} \\
(r, \theta, z)\end{array}$ & $\begin{array}{l}\text { Reynolds number }[-] \\
\text { cylindrical coordinate system }[(m, m, m)]\end{array}$ & & wall shear stress $\left[\mathrm{m}^{2} / \mathrm{s}^{2}\right]$ \\
\hline
\end{tabular}

\section{Method}

In this paper, the flow in an infinite square array of infinitely long cylinders is modelled with a single-cylinder geometry. As the array is assumed to be infinitely wide and long, both array boundary and flow entrance effects are neglected. The fluid is incompressible, Newtonian and isothermal. Multiple cases are computed using Large-Eddy Simulations in OpenFOAM ${ }^{\circledR}$. Subsequently, a spectral analysis is performed on the cylinder wall pressure profile, after which these frequency spectra are autocorrelated. The influence of the cylinder spacing on the cylinder wall pressure spectrum is analyzed.

\subsection{Flow domain}

The flow domain consists of a rectangular channel located around a single cylinder, as shown in Figure 3a. This cross-section can be considered as a unit cell for the square array shown in Figure 2. Due to the absence of entrance or array boundary effects, there are only two geometrical parameters of importance: the cylinder diameter $D$ and the pitch $P$. The latter is the distance between the centres of two adjacent cylinders. A no-slip condition is applied on the 
rigid and stationary cylinder wall, represented by the thick black line in Figure 3a. Periodic boundary conditions are enforced on the outer domain limits: the boundaries which are periodic are depicted in the same color in Figure 3a. The in- and outlet are located on the bottom and top side of the geometry shown in Figure $3 \mathrm{~b}$. The in- and outlet conditions are also periodic, but with a prescribed pressure gradient in the axial direction.

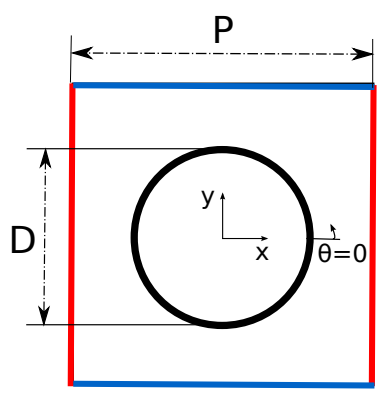

(a)

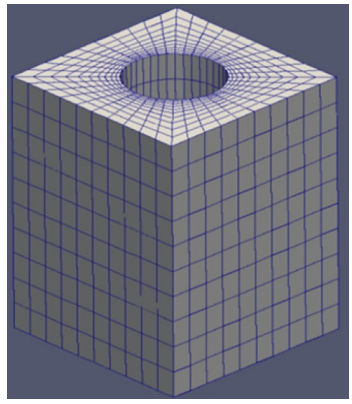

(b)

Figure 3: (a) Schematic top view of the flow domain. The pitch $P$ and diameter $D$ are shown. The boundaries of the same color are periodic. The thick black line denotes the cylinder wall. The $\mathrm{x}$ - and $\mathrm{y}$-axis and the reference $\theta$ equal to $0^{\circ}$ are also shown. (b) View of a coarse mesh on the $5 \%$ upper part of the domain.

\subsection{Non-dimensional variables}

Before going into the details of the numerical analysis, the relevant nondimensional parameters are identified. The mean axial flow speed $U$ is made non-dimensional by defining the Reynolds number:

$$
R e_{D_{h}}=\frac{\rho U D_{h}}{\mu}=\frac{U D_{h}}{\nu},
$$

where $\rho$ is the fluid density and $\mu$ and $\nu$ denote the dynamic and kinematic viscosity, respectively. Assuming infinitely long cylinders, the only relevant geometrical parameters are the pitch $P$ and the diameter $D$. The $P / D$-ratio is the non-dimensional parameter related to the cylinder spacing. However, the finite length of the numerical domain has some influence on the results, as will be discussed in Section 3. The domain length $L$ is made non-dimensional by dividing it by the hydraulic diameter $D_{h}$, which is equal to four times the through-flow area divided by the wall perimeter in the domain:

$$
D_{h}=4 \frac{P^{2}-\frac{\pi}{4} D^{2}}{\pi D} .
$$

The frequency of the pressure fluctuations is made non-dimensional by multiplication with the ratio of the hydraulic diameter and the mean axial flow speed, giving the following equation for the Strouhal number:

$$
S t=\frac{f D_{h}}{U} \text {. }
$$




\subsection{Large-Eddy Simulation}

In Large-Eddy Simulations (LES), the large eddies are resolved and the smallest vortices, which are universal in nature according to Kolmogorov's hypothesis, are modelled. The small-scale turbulent eddies are modelled with a stress tensor - called the subgrid-scale stress - in the momentum equation. The direct calculation of the large vortices causes the computational load to be considerably higher compared to URANS.

The sub-grid scale stress model applied in this thesis is the Smagorinsky model with dynamic Lagrangian averaging (Meneveau et al. (1996)). Firstly, the tophat filtering function $G\left(x, x^{\prime}, \bar{\Delta}\right)$ is defined as follows:

$$
G\left(x, x^{\prime}, \bar{\Delta}\right)= \begin{cases}\frac{1}{\bar{\Delta}}, & \left|x-x^{\prime}\right| \leq \frac{\bar{\Delta}}{2} \\ 0, & \text { elsewhere }\end{cases}
$$

where the filter width $\bar{\Delta}$ is equal to the geometric average of the cell width in the three dimensions: $\bar{\Delta}=\left(h_{x} h_{y} h_{z}\right)^{1 / 3}$.

The filtering function is multiplied with a field variable $f$ and subsequently integrated over the entire domain. This filtering operation is applied to the Navier-Stokes equations for an incompressible, isothermal, Newtonian fluid. A filtered variable $f$ is denoted by $\bar{f}$. The flow equations become:

$$
\begin{gathered}
\frac{\partial \overline{u_{i}}}{\partial x_{i}}=0 \\
\frac{\partial \overline{u_{i}}}{\partial t}+\frac{\partial}{\partial x_{j}}\left(\overline{u_{i}} \overline{u_{j}}\right)=-\frac{1}{\rho} \frac{\partial\left(\bar{p}+\overline{p_{A}}\right)}{\partial x_{i}}-\frac{\partial \tau_{i j}}{\partial x_{j}}+\nu \frac{\partial^{2} \overline{u_{i}}}{\partial x_{i} \partial x_{j}}
\end{gathered}
$$

where $\tau_{i j}$ is the residual stress, given by:

$$
\tau_{i j}=\overline{u_{i} u_{j}}-\overline{u_{i}} \overline{u_{j}} .
$$

An additional pressure term $\overline{p_{A}}$ is inserted into the momentum equation in order to achieve the desired mean axial flow speed. The filtered variables $\bar{u}$ and $\bar{p}$ are calculated directly by solving the filtered Navier-Stokes equations, which requires an appropriate model for the residual stress $\tau_{i j}$. The Smagorinsky model yields, with the Einstein notation:

$$
\begin{gathered}
\tau_{i j}-\frac{\delta_{i j}}{3} \tau_{k k}=-2 \nu_{t} \overline{S_{i j}} \\
\nu_{t}=\left(C_{S} \bar{\Delta}\right)^{2}|\bar{S}|
\end{gathered}
$$

where $\overline{S_{i j}}=\frac{1}{2}\left(\frac{\partial \overline{u_{i}}}{\partial x_{j}}+\frac{\partial \overline{u_{j}}}{\partial x_{i}}\right)$ and $|\bar{S}|=\left(2 \overline{S_{i j}} \overline{S_{i j}}\right)^{1 / 2}$.

$C_{S}$ is the Smagorinksy constant. This parameter is updated with information extracted from the filtered variables. The latter is done by defining another filtering function, the test filter, based on a filter width $\widehat{\Delta}: \widehat{\Delta}=2 \bar{\Delta}$. A variable $f$ which is filtered with the test filter, is denoted by $\widehat{f}$. The Smagorinsky model parameter is calculated with the Lagrangian dynamic model given by Equation (9).

$$
C_{S}^{2}(x, t)=\frac{f_{L M}(x, t)}{f_{M M}(x, t)}
$$


The quantities $f_{L M}$ and $f_{M M}$ are calculated from two additional transport equations (Meneveau et al. (1996)). The applied discretisation scheme is the standard Gaussian finite volume integration. The pressure velocity coupling was done with the PISO-algorithm and the standard geometric-algebraic multigrid solver in OpenFOAM was used. Time stepping is done with the standard backward Euler scheme.

\subsection{Spectral analysis}

After the LES simulation in OpenFOAM, the pressure profile on the cylinder wall is postprocessed. The wall pressure is dependent on two spatial dimensions - the axial $(z)$ and circumferential $(\theta)$ coordinate on the cylinder wall - and on the time variable $n$. $\theta$ is set equal to $0^{\circ}$ in a gap region, which means that the subchannel regions adjacent to it are designated by $\theta=45^{\circ}$ or $-45^{\circ}$. All multiples of $90^{\circ}$ also correspond to a gap region.

First, the temporal Discrete Fourier Transform is calculated with Equation (10). The number of time steps considered is denoted by $N$, the duration of 1 time step is $\Delta t$. The frequency is expressed with the Strouhal number.

$$
P(\theta, z, S t)=\sum_{n=0}^{N-1} p(n) \exp \left(-j \frac{2 \pi n k}{N}\right)
$$

Applying Equation (3) yields $S t=\frac{k}{N \Delta t} \frac{D_{h}}{U}$.

The obtained Fourier variables $P$ are substituted into a spatial autocorrelation, called the Cross Spectral Density (CSD), given by:

$C S D\left(\theta_{1}, \theta_{2}, \Delta z, S t\right)=\frac{\Delta t}{2 N N_{z}} \sum_{m=0}^{3} \sum_{z=0}^{N_{z}-1} P^{*}\left(\theta_{1}+m \frac{\pi}{2}, z, S t\right) P\left(\theta_{2}+m \frac{\pi}{2}, z+\Delta z, S t\right)$.

The Power Spectral Density (PSD) is a special case of the CSD: $P S D(\theta, S t)=$ $C S D(\theta, \theta, 0, S t)$. Both quantities are made non-dimensional: the PSD is divided by the constant $\rho^{2} U^{3} D_{h}$ and the CSD is converted to the coherence function $\gamma$ :

$$
\gamma\left(\theta_{1}, \theta_{2}, \Delta z, S t\right)=\frac{C S D\left(\theta_{1}, \theta_{2}, \Delta z, S t\right)}{\operatorname{PSD}\left(\theta_{1}, S t\right)} .
$$

As the noise on the CSD is rather large, an averaging procedure is applied: the total time batch is divided into several parts and the CSD is calculated for each part separately, after which the global CSD is found by taking the average of the computed CSD profiles. However, one should take into account that this procedure affects the frequency limitation of the CSD due to the finite summation in the CSD formulation. The lowest frequencies (near to zero) are merely a numerical residu of the calculation. Decreasing the size of the summation, e.g. by splitting the time batch in smaller parts, increases the minimum frequency above which the CSD has a physical meaning. This limitation is checked in 
Section 4 by calculating the theoretical minimal frequency obtainable with the CSD calculations as follows:

$$
f_{\text {min }}=\frac{1}{N_{\text {eval }} \Delta t} \Leftrightarrow S t_{\text {min }}=\frac{f_{\text {min }} D_{h}}{U}=\frac{D_{h}}{N_{\text {eval }} \Delta t U},
$$

where $N_{\text {eval }}$ and $\Delta t$ represent the number of considered time steps and the time step size respectively.

\section{Grid convergence analysis}

In order to determine the sensitivity of the solution on the grid, different meshes are constructed for a constant flow domain. The reference geometry has a $P / D$-ratio equal to 1.085 and an $L / D_{h}$-ratio of 15 . The grid convergence study is done with a Reynolds number $\operatorname{Re}_{D_{h}}$ equal to 14,000 . The different cases are listed in Table 1. The simulation results are shown in Figure 4. All

figures in this paragraph contain non-dimensional variables. The wall profile in the subchannel region is similar for all the computed cases.

Table 1: Cases with different grid properties applied on the reference flow domain in order to determine the grid convergence.

\begin{tabular}{|l|c|c|c|c|}
\hline Case & $\Delta x^{+}[-]$ & $y^{+}[-]$ & $\Delta z^{+}[-]$ & Number of cells [-] \\
\hline X50 & 100 & 2 & 20 & $2,620,800$ \\
X30 & 60 & 2 & 20 & $4,368,000$ \\
X20 & 40 & 2 & 20 & $6,552,000$ \\
Y06 & 100 & 1.2 & 20 & $3,307,200$ \\
Z06 & 100 & 2 & 12 & $4,233,600$ \\
\hline
\end{tabular}

Figure $4 \mathrm{~b}$ shows that changing the $\Delta x^{+}$-value alters the PSD significantly, but not in the Strouhal number range between 0 and 0.4, which is the relevant Strouhal number range in this paper. In this region of interest, the PSD profiles are similar for all cases. For higher Strouhal numbers, it is argued that the X50 case is the most precise case because it has the finest mesh and thus allows the calculation of smaller eddies than the X20 and X30 case. As the refined meshes in cases Y06 and Z06 do not yield significantly different results compared to case $\mathrm{X} 50$, the resolution of case X50 is applied in the remainder of this paper. The correlation used as a benchmark in this figure (red curve) is Curling's correlation (Curling and Païdoussis (1992)). Additionally, Piomelli (2012) states that the following range is appropriate for the mesh:

$$
\begin{aligned}
\Delta x^{+}=\frac{\Delta x}{y_{\tau}} & =15-40 \\
y^{+} & =\frac{y}{y_{\tau}}<1 \\
\Delta z^{+}=\frac{\Delta z}{y_{\tau}} & =50-150
\end{aligned}
$$


in which $\Delta x, y$ and $\Delta z$ denote the cell spacing along the cylinder circumference, the radial height of the first cell next to the cylinder wall and the cell spacing in the axial direction, respectively. The wall shear stress $\tau_{w}$, expressed in $\mathrm{m}^{2} / \mathrm{s}^{2}$, is used to define the velocity $U_{\tau}=\sqrt{\tau_{w}}$. $y_{\tau}$ is subsequently defined as $y_{\tau}=\nu / U_{\tau}$. It was verified that the conditions in Equation (14) were met for all cases in Table 3.

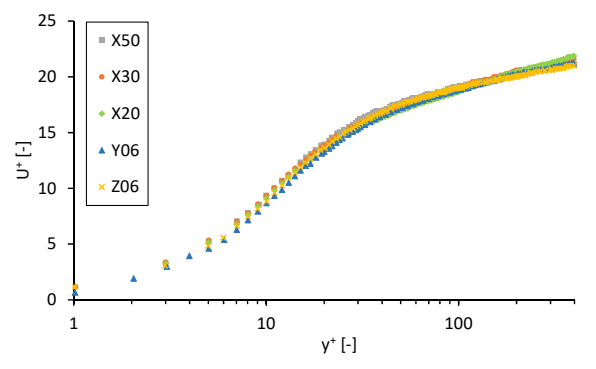

(a)

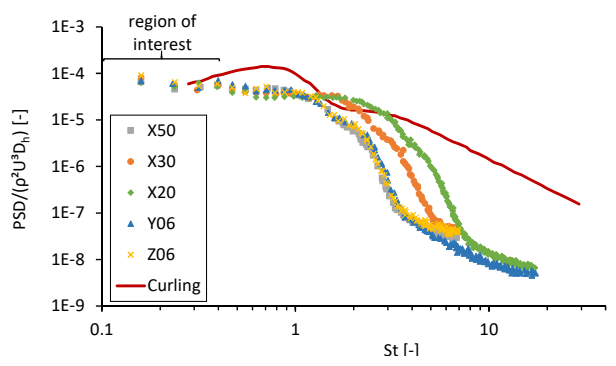

(b)

Figure 4: Results of the grid sensitivity analysis. The profiles are time-averaged values for a point on the cylinder wall located in the subchannel region $\left(\theta=45^{\circ}\right)$. (a) Wall velocity profile (b) $\operatorname{PSD}\left(45^{\circ}, \mathrm{St}\right)$ obtained from LES calculations and Curling's correlation (Curling and Païdoussis (1992)).

Additionally, the influence of the domain length on the PSD is investigated. Two cases containing a single cylinder with the same cylinder diameter and pitch, but with different cylinder length, are defined in Table 2 and the results are shown in Figure 5. The conclusion is that a domain length of 15 times the hydraulic diameter is sufficient not to have an influence of the domain length on the PSD profile in the subchannel region. In the simulations presented further in this paper, the $L / D_{h}$-ratio is above 15 in most cases, although the lowest $L / D_{h}$-ratio is equal to 9 . This is deemed to give acceptable results, as these results will be compared with literature. Curling's correlation does not follow the present simulation data, especially at Strouhal numbers above 2. This is possibly due to the level of turbulence in the experiments by Curling which is presumably higher than in the LES calculations shown here.

Table 2: Cases defined to analyze the sensitivity on the domain length. The cylinder has a diameter equal to $0.2 \mathrm{~m}$ and a pitch of $0.217 \mathrm{~m}$. The hydraulic diameter $D_{h}$ equals $0.1 \mathrm{~m}$.

\begin{tabular}{|l|c|c|}
\hline Case & $L / D_{h}[-]$ & Number of cells [-] \\
\hline$L / D_{h}=15$ & 15 & $2,620,800$ \\
$L / D_{h}=30$ & 30 & $5,241,600$ \\
\hline
\end{tabular}




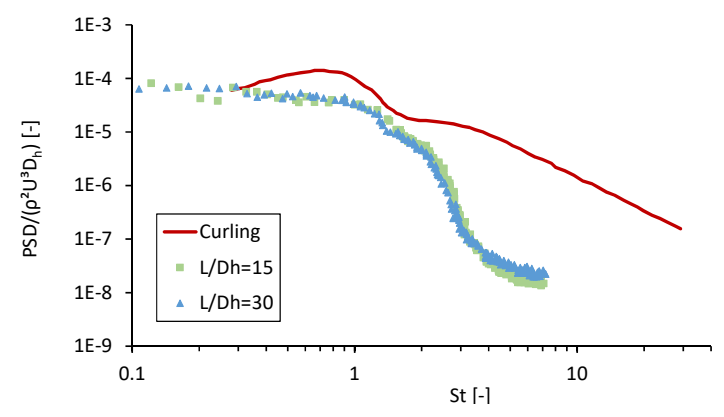

Figure 5: Effect of the domain length on $\operatorname{PSD}\left(\theta=45^{\circ}, \mathrm{St}\right)$. The profiles are average values for a point on the cylinder wall located in the subchannel region. The compared cases have a $L / D_{h}$-ratio of 15 and 30 , respectively.

\section{Analysis of the PSD}

Important features of the flow can be extracted from the PSD, such as the location and frequency of the dominant pressure fluctuations. Consequently, the first complete flow analysis is aimed at the PSD only. The numerical simulations on which this analysis relies are performed on different cases, which are listed in Table 3. The Reynolds number $R e_{D_{h}}$ is 10,000 in all simulations and the fluid is water. As a result, the mean axial flow velocity $U$ is adapted according to the chosen cylinder spacing, i.e. $P / D$-ratio, which differs for all the defined cases. The used time step is denoted by $\Delta t$. This variable is made non-dimensional by multiplication with the ratio $U / \Delta z$. Not all time steps are taken into consideration in the subsequent spectral analysis: it is well known that the simulation requires some time steps to get physical turbulence (Piomelli (2012)). Also, the first time steps of every calculation are influenced by the initialization of the flow and therefore have to be omitted from further analysis. It was checked for all cases that regime conditions were achieved. This occurred after having calculated between 2 and 5 through-flow times. Consequently, the number of time steps $N_{\text {eval }}$ used in the spectral analysis is different for every case, as given in Table 3.

Considering the discussion in Section 2, the minimal Strouhal number $S t_{\text {min }}$, which is defined in Equation 13, is calculated for all the cases defined in Table 3. Case $P / D=1.2$ yields the highest value for $S t_{\min }$, i.e. 0.006 . The noise in the PSD-graph remains however high even for Strouhal numbers above this minimal value. Additionally, the PSD is periodic over $90^{\circ}$ due to the flow domain's periodicity. The PSD's periodicity has been verified with the present simulations. The relative error on the PSD made by assuming periodicity for the Strouhal number up to 4 , equals $9.0 \%$ in case $P / D=1.06$ and $4.3 \%$ in case $P / D=1.2$. This error was defined as the average absolute difference of the local PSD value with the periodic PSD value, divided by the latter. Taking into account the noise on the PSD, these errors are small and thus periodicity over $90^{\circ}$ can be assumed safely in the subsequent analysis. In the first part of this 
section, a general overview of the PSD will be given. Subsequently, it will be discussed to what extent the observed features depend on the Reynolds number and on the $P / D$-ratio.

Table 3: Non-dimensional characteristics of the cases computed to analyze the influence of the $P / D$-ratio on the PSD. For all cases, $R e_{D_{h}}$ equals 10,000 .

\begin{tabular}{|c|c|c|c|c|c|}
\hline Case & $\mathrm{P} / \mathrm{D}$ & $L / D_{h}$ & $\Delta t U / \Delta z$ & $N_{\text {eval }}$ & Cells \\
\hline$P / D=1.02$ & 1.02 & 23 & 0.063 & 29,220 & $4,800,000$ \\
$P / D=1.03$ & 1.03 & 21 & 0.030 & 52,620 & $4,800,000$ \\
$P / D=1.04$ & 1.04 & 20 & 0.027 & 67,884 & $4,800,000$ \\
$P / D=1.06$ & 1.06 & 17 & 0.231 & 20,672 & $4,800,000$ \\
$P / D=1.085$ & 1.085 & 15 & 0.268 & 48,000 & $4,800,000$ \\
$P / D=1.15$ & 1.15 & 11 & 0.196 & 54,720 & $6,400,000$ \\
$P / D=1.2$ & 1.2 & 9 & 0.160 & 50,000 & $3,072,000$ \\
$P / D=1.3$ & 1.3 & 13 & 0.112 & 20,733 & $11,520,000$ \\
$P / D=1.4$ & 1.4 & 10 & 0.088 & 8,973 & $11,520,000$ \\
\hline
\end{tabular}

\subsection{General view of the PSD}

The primary features of the PSD will be investigated by using the simulation results from two cases defined in Table 3 : cases $P / D=1.06$ and $P / D=1.2$. The PSD graphs for these cases are shown in Figures 6 and 7 . The PSD is made non-dimensional by dividing it with $\left(\rho^{2} U^{3} D_{h}\right)$. All figures in this paragraph are made non-dimensional, unless specified otherwise. Note that the graphs differ substantially: very sharp peaks are found for case $P / D=1.06$, whereas a smoother profile is obtained for case $P / D=1.2$. It is expected that this is related to the relative strength of the flow instability compared to the turbulent content of the flow. On the one hand, an instability exists because of the flow speed difference between the gap and the subchannel region, creating periodic pressure fluctuations. It is therefore understandable that sharp peaks are located at the dominant frequencies corresponding to these fluctuations. On the other hand, turbulence is a non-periodic phenomenon and consequently causes a break-up of the periodic instability. As a result, the periodic behaviour decays quickly when the turbulence become more dominant. Apparently, the instability is dominant for case $P / D=1.06$ whereas the turbulence is more important for case $P / D=1.2$. In conclusion, the instability is strong for small $P / D$-ratios only, whereas it is only of minor importance when the space between the cylinders becomes larger.

For a Reynolds number of 10,000 , sharp peaks are present up to a $P / D$ ratio of 1.085, below which the general look of the PSD is similar to the one depicted in Figure 6. It was not investigated at which $P / D$-ratio the PSD obtains a smoother profile, but this is certainly the case for $P / D$ larger than 1.2. As the presence of these peaks is related to the relative importance of the Kelvin-Helmholtz instability compared to the turbulence, it is expected that 


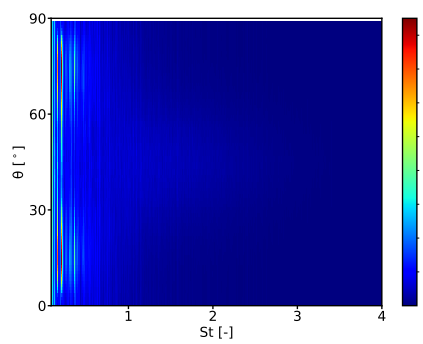

(a)

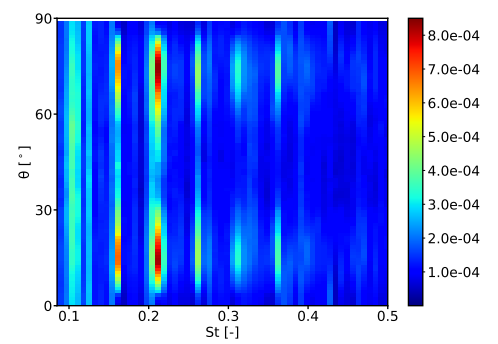

(b)

Figure 6: PSD for case $P / D=1.06$, as a function of $\theta$ and Strouhal number. (a) Global view (b) Zoom at small $S t$

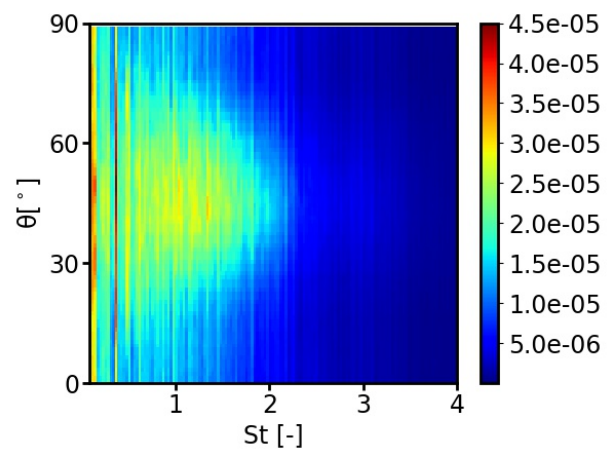

Figure 7: PSD for case $P / D=1.2$, as a function of $\theta$ and Strouhal number. Note the difference in color scale compared to Figure 6. 
the continuous profile is favoured for higher Reynolds numbers, which will be discussed next.

\subsection{Influence of the Reynolds number}

The PSD characteristics do not change significantly with the Reynolds number as long as the flow remains turbulent everywhere (Lexmond et al. (2005)). However, laminarisation cannot be prevented in (parts of) the flow domain at a Reynolds number $R e=10,000$, especially considering that the local Reynolds number in the gap is lower. It is therefore not surprising that the Reynolds number affects the PSD and CSD graphs. This is shown in Figure 8, depicting the PSD as a function of $\theta$ and the Strouhal number for $P / D=1.085$ and for two different Reynolds numbers. At a Reynolds number of 15,000 , a maximum occurs at a lower Strouhal number than for Reynolds number 10,000 (0.14 and 0.18 compared to 0.2 and 0.27 , respectively). This shift is related to the Strouhal number definition: the denominator contains $U$, which is 1.5 times as large for the high Reynolds number case as it is for the low Reynolds number case. Additionally, the non-dimensional PSD values are lower for $R e=15,000$ than for $R e=10,000$. This is influenced by the denominator of the non-dimensional PSD, which is considerably larger for the high Reynolds number case. The dimensional PSD increases with increasing Reynolds number: the higher flow velocity and higher turbulent intensity provoke stronger pressure fluctuations. Abbasian et al. (2010) performed LES calculations on a circular flow channel containing four rods and eight half-rods and showed that the amplitude of the pressure oscillation grows in the gap region for increasing Reynolds number (for a $P / D$-ratio equal to 1.08 ), thereby confirming that the dimensional PSD is higher for the higher Reynolds number.

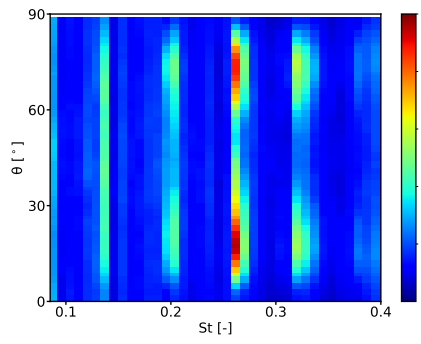

(a)

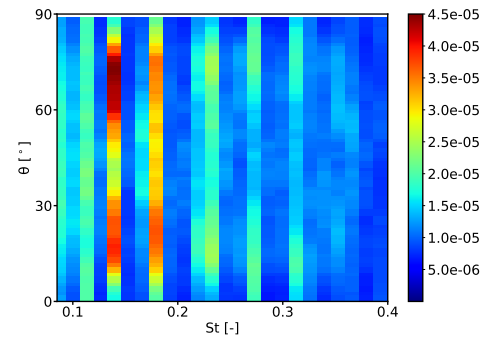

(b)

Figure 8: $\operatorname{PSD}(\theta, S t)$ for $P / D=1.085$. Note the difference in color scale between both graphs. (a) $R e=10,000$ (b) $R e=15,000$

In the simulations described in following sections, the Reynolds number is equal to 10,000 . The Reynolds number is kept low to limit computational resources and it also has the beneficial effect that the influence of turbulence on 
the flow is limited. However, this does not mean that the Kelvin-Helmholtz instability disappears at higher Reynolds numbers. Merzari et al. (2008) showed that the coherent structures are still present at a Reynolds number of 38,754 with $P / D=1.06$.

\subsection{Influence of the cylinder spacing}

The previous section already indicates that the cylinder spacing strongly affects the coherent flow structures. For very large $P / D$-ratios, the cylinder cluster effectively reduces to independent cylinders, unaffected by the distant presence of their neighbours. For smaller cylinder spacings, the flow field changes due to the difference between gap and subchannel regions, leading to the occurrence of a Kelvin-Helmholtz instability. The characteristics of the cylinder wall pressure fluctuations change substantially. In this section, the values and the location of the pressure maxima and the frequency content of the wall pressure field are analyzed by means of the PSD. The characteristics of the cases used in this analysis are listed in Table 3 .

\subsubsection{Maximum amplitude}

The PSD amplitude is a direct measure for the strength of the cylinder wall pressure fluctuations or, similarly, for the strength of the Kelvin-Helmholtz instability in the flow. For each case in Table 3, the maximal value of the PSD is determined. Figure 9a shows the maximal value of the PSD as a function of the $P / D$-ratio of the corresponding case. The logarithmic scale on the vertical axis allows the visualization of the rapid decrease of the PSD maximum at higher cylinder spacings. It should be noted that the noise on the PSD signal is large. Therefore, the algebraic average of the PSD over the entire range of the circumferential coordinate $\theta$ at the Strouhal number at which the local PSD maximum arises is calculated -rather than only taking the average in the axial direction, as given in Equation 11. This corresponds to the average value over a vertical line located at the specified Strouhal number in Figure 8a. The result is given in Figure 9b. It is noted that the overall trend is similar in both cases, meaning that the noise on the local PSD value does not significantly influence the qualitative trend.

Figure 9 shows a strong decrease of the PSD maximum for increasing cylinder spacing, at least for $P / D$ larger than 1.03. The decrease is nearly exponential. For example, the maximal value for the PSD at $P / D$ equal to 1.4 is a factor $10^{4}$ smaller than in the case of $P / D$ equal to 1.2 . This indicates that the Kelvin-Helmholtz instability is barely present for the geometries with the largest cylinder spacing in this analysis. It is noted that the maximal transverse velocity component has a magnitude of the order of $0.0002 \mathrm{~m} / \mathrm{s}$, whereas the mean axial flow velocity for case $P / D=1.4$ equals $33.43 \mathrm{~m} / \mathrm{s}$. This indicates that the Kelvin-Helmholtz instability will have a negligible influence on the PSD profile. As mentioned before, the turbulence field was not fully developed for the last two cases in Table 3.

The driving force behind the Kelvin-Helmholtz instability is the axial flow velocity difference between the gap and subchannel region. The distinction 


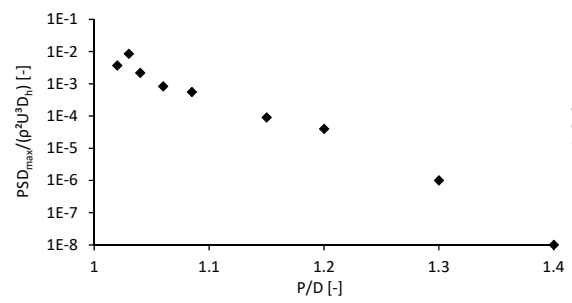

(a)

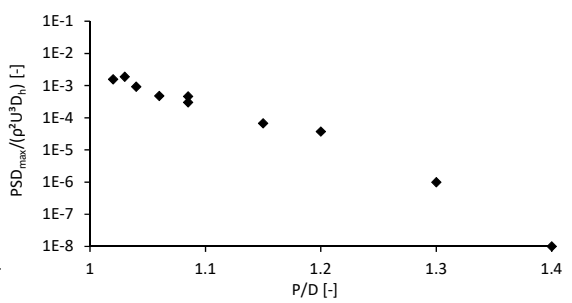

(b)

Figure 9: Variation of the PSD maximum as a function of $P / D$. The PSD is calculated as a mean value over the entire range of the axial coordinate. (a) PSD maximum located at a single circumferential position on the cylinder wall (b) PSD maximum averaged over the circumferential direction, at the same Strouhal number as the local maximum

between both subregions becomes increasingly smaller for increasing cylinder spacing, which explains the rapid decrease of the PSD maximum for $P / D$ larger than 1.03. However, the PSD maximum for $P / D$ equal to 1.02 is significantly smaller than for 1.03. The reason for this behaviour is the following: the KelvinHelmholtz instability results in cross-flow between one subchannel through a neighbouring gap region towards another subchannel. The viscous forces, mainly present in the gap region due to the vicinity of the wall, inhibit this fluid motion. For an increasingly smaller gap between the cylinders, the friction in the gap becomes more important, therefore reducing the cross-flow speed and thus the pressure fluctuation amplitude. The resulting graph of the PSD maximum as a function of the $P / D$-ratio is thus the result of two phenomena: the viscous forces in the gap region inhibiting the cross-flow through the gap and the (axial) flow velocity difference between the gap and subchannel region, the source of the flow instability. It is remarkable that Yan and Yu (2011), who performed URANS simulations on a periodic element of a hexagonal array at a Reynolds number of 38,754 , also find that the pressure fluctuations are maximal at $P / D=1.03$.

To conclude this discussion, a correlation which relates the PSD maximum to the $P / D$-ratio, is proposed in Equation (15). The data from Figure $9 \mathrm{~b}$ are reproduced in Figure 10, on which the new correlation is superimposed. The most accurate fit to the results presented in Figure 9 was obtained with a piecewisedefined function. For $P / D$ smaller than 1.03, a linear function was determined by applying a least-squares model to three datapoints: for $P / D$ equal to 1 - at which the PSD due to the Kelvin-Helmholtz instability is assumed to be zero 1.02 and 1.03. For larger gap sizes, an exponential function provided the best fit. It is noteworthy that the constant in the exponential is rather large: around 30. As the noise on the PSD found in literature is large (Païdoussis (1998)), there is some uncertainty about this value.

$$
\frac{P S D_{\max }}{\rho^{2} U^{3} D_{h}}= \begin{cases}0.06550\left(\frac{P}{D}\right)-0.06550, & 1 \leq \frac{P}{D} \leq 1.03 \\ 6.89110^{10} \exp \left(-30.28\left(\frac{P}{D}\right)\right), & 1.03<\frac{P}{D}\end{cases}
$$




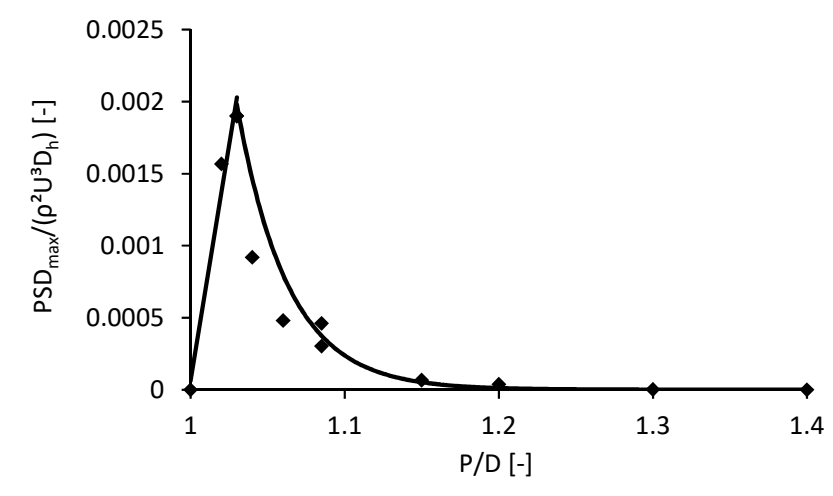

Figure 10: Variation of maximum of the circumferentially averaged PSD as a function of $P / D$. The curve is given by Equation (15).

\subsubsection{Circumferential position of the PSD maximum}

Due to the geometrical periodicity, the PSD-graph is periodic in $\theta$. Therefore, only values of $\theta$ within the range of $\left[0^{\circ}-90^{\circ}\right]$ are shown. The local PSD maximum - the value shown in Figure 9a - is found on a well-determined circumferential coordinate on the cylinder wall. Figure 11 shows the circumferential position at which the maximal value of the PSD is located. There are two maxima, one at an angle $\theta_{1, \max }$ smaller than $45^{\circ}$ and another one at an angle $\theta_{2, \max }$ larger than $45^{\circ}$. For almost all data points, $\theta_{1, \max }+\theta_{2, \max }=90^{\circ}$, indicating symmetry with respect to the subchannel region. Secondly, the angle is constant for low $P / D$-ratios, up to $P / D=1.06$. In this case, $\theta_{1, \max }$ and $\theta_{2, \max }$ become $13^{\circ}$ and $77^{\circ}$, respectively. The pressure fluctuations along the cylinder are thus stronger close to the gap region than to the subchannel region, which is probably related to the fact that the cross-flow is strongest in the gap region. However, the influence of the viscous forces on the flow is also strongest around $0^{\circ}$. The obtained limit of $\theta_{1, \max }$ equal to $13^{\circ}$ is the result of these two influences. For higher $P / D$-ratios, and thus a decreasingly strong instability, the angles come closer together. This is due to the relatively stronger turbulence: in case there were only turbulent fluctuations, the PSD maximum would be located at $45^{\circ}$. This is due to the slightly higher flow velocity in the subchannel region compared to the gap region. As the local Reynolds number is higher in the subchannel region, the turbulent fluctuations and thus the pressure oscillations are strongest in the subchannel region. When increasing the cylinder spacing even further, the distinction between the gap and the subchannel disappears and the PSD is homogeneous over the entire frequency range and for all angles $\theta$.

Another remark about Figure 11 is in order. The graph only shows the position along the cylinder circumference of the absolute PSD maximum for every case. However, there is a smaller local maximum which is located at $45^{\circ}$ and at a higher Strouhal number for $P / D=1.15$ and $P / D=1.2$. This is shown 


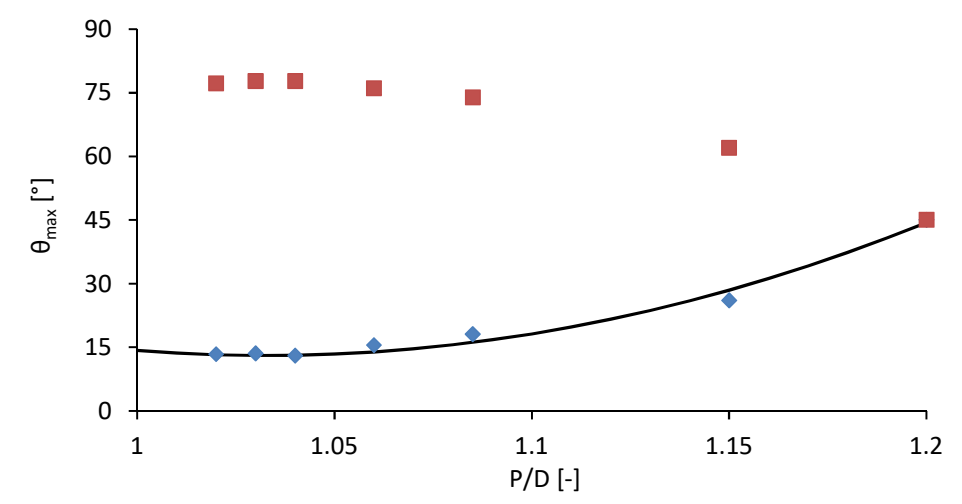

Figure 11: Variation of the circumferential position of the PSD maximum as a function of $P / D$ (blue and red dots). The line connecting the blue datapoints is given by Equation (16).

in Figure 7. The local maximum located around $\theta$ equal to $45^{\circ}$ is provoked by turbulence, of which the level is highest in the subchannel region. Therefore, the graphs confirm that turbulence becomes more dominant with respect to the instability for increasing cylinder spacings. The presence of a local PSD maximum around $45^{\circ}$ is expected to occur if $P / D \geq 1.15$. At some point, the turbulence effects outweigh the coherent structures, giving rise to an absolute maximum around $45^{\circ}$. This happens for $P / D>1.2$ and as long as there is a noticeable difference between gap and subchannel regions, as previously mentioned.

Lastly, a least squares fit is applied to the data in Figure 11. This yields the following result, which is valid until $P / D=1.2$ :

$$
\theta_{\max }=19.5\left(\frac{P}{D}\right)^{2}-40.275\left(\frac{P}{D}\right)+21.02[\mathrm{rad}]
$$

in which $\theta_{\max }$ is expressed in radians and obtains the value of $\pi / 4 \mathrm{rad}$ or $45^{\circ}$ when $P / D$ equals 1.2 . For cylinder spacings beyond $P / D=1.2$, the PSD maximum is still located at $45^{\circ}$ until the cylinders are placed so far apart that the distinction between gap and subchannel region disappears, at which point a homogeneous PSD is expected.

\subsubsection{Frequency corresponding to the PSD maximum}

It is observed that the PSD maximum does not occur at a universal Strouhal number value. Instead, the Strouhal number $S t_{\max }$ at which the PSD maximum occurs, is different for each computed case. Figure 12 shows the variation of $S t_{\max }$ as a function of the cylinder spacing (red squares). The function is peculiar: the exact behaviour of the Strouhal number $S t_{\max }$ is not clear. The function appears to decrease with increasing $P / D$ for low $P / D$-ratios and goes through a minimum at $P / D=1.04$. However, the dimensional frequency 
$f_{\max }=S t_{\max } U / D_{h}$ (not shown) is monotonously decreasing with increasing $P / D$.

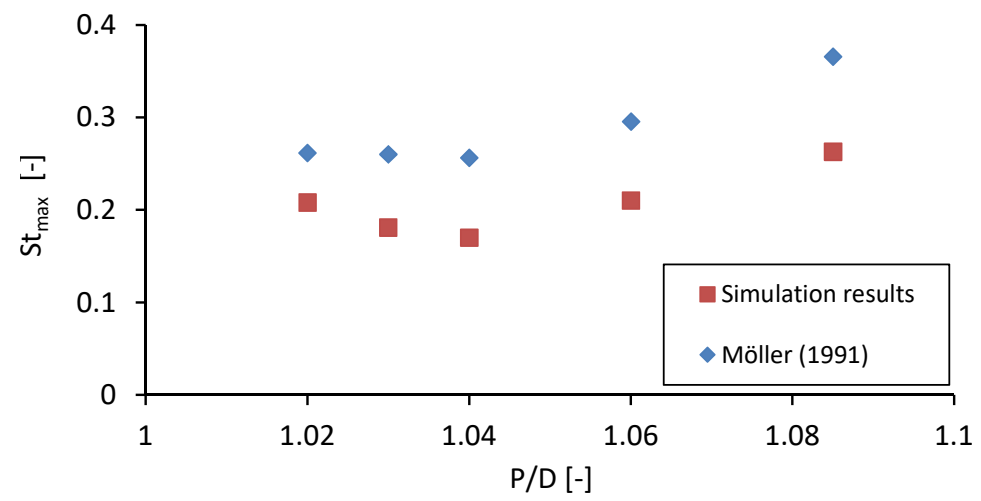

Figure 12: Variation of the Strouhal number at which the PSD maximum occurs $S t_{\max }$, according to the present simulations (red squares) and Möller's correlation (blue diamonds), respectively.

Möller (1991) investigated axial air flow through a rectangular channel containing four cylindrical rods placed side by side and proposed Equation (17) to quantify the influence of the cylinder spacing on the Strouhal number corresponding to the maximum PSD. However, care should be taken when interpreting these results. Möller defines the Strouhal-number in a different way than in this paper, i.e. based on the cylinder diameter $D$ and the velocity $U_{\tau}=\sqrt{\tau_{w}}$. Also, this correlation was developed with empirical data of flows with higher Reynolds numbers -between 20,000 and 200,000- and therefore includes a larger effect of turbulence.

$$
\left(S t_{u_{\tau}}\right)^{-1}=\left(\frac{f D}{\sqrt{\tau_{w}}}\right)^{-1}=0.808 \frac{P-D}{D}+0.056
$$

The wall shear stress $\tau_{w}$ is computed from the force equilibrium between the pressure gradient over the domain length and the wall shear stress:

$$
\begin{gathered}
A_{\text {flow }} \Delta p=A_{\text {wall }} \rho \tau_{w} \Rightarrow\left(P^{2}-\frac{\pi}{4} D^{2}\right) \Delta p=(\pi D L) \rho \tau_{w} \\
\Leftrightarrow \tau_{w}=\frac{\Delta p}{\rho} \frac{P^{2}-\frac{\pi}{4} D^{2}}{\pi D L}
\end{gathered}
$$

Möller's correlation, adapted to the Strouhal number definition used in this paper, is compared to the present LES results in Figure 12. It is concluded that the overall trend is similar for both the Large-Eddy Simulations (red squares) and Möller's correlation (blue diamonds), but the latter predicts consistently higher values for the Strouhal number at which the PSD maximum occurs. The discrepancy between both could be due to the numerical error on $\tau_{w}$, obtained 
from LES, as well as due to the low Reynolds number at which the simulations in this paper were performed. However, it is more probable that the finite domain length influences this result. A characteristic frequency $f_{D L}$ and corresponding Strouhal number $S t_{D L}$, related to the domain length $L$, is defined as follows:

$$
\begin{gathered}
f_{D L}=\frac{U_{c}}{L}, \\
S t_{D L}=\frac{f_{D L} D_{h}}{U}=\frac{U_{c} D_{h}}{U L} .
\end{gathered}
$$

In Equation (19), the characteristic speed is the convection speed $\mathrm{U}_{\mathrm{c}}$, which is the speed at which the periodic instability is transported through the flow. $U_{c}$ is considered to be more appropriate as a reference velocity than the average flow speed $U$ because it is more closely related to the physics of the occurring eddies than $U$. Moving ahead of the analysis in Section 5, the convection speed can be estimated with an adapted version of the correlation defined by Guellouz and Tavoularis (2000):

$$
\frac{U_{c}}{U}=1.11\left(1-\exp \left(-17 \frac{P}{D}+16.3\right)\right)
$$

Comparing the Strouhal number values shown in Figure 12 with the value of $S t_{D L}$ for each $P / D$-ratio, it is concluded that the former is close to ${ }^{1}$ a discrete multiple of the latter in five of the seven cases (not shown). This leads to the conclusion that the domain length probably influences the frequency content of the PSD, a hypothesis which will be reinforced in Section 4.3.4. This is due to the periodic boundary conditions at the in- and outlet of the domain: these boundary conditions force a discrete number of large eddies into the domain, therefore artificially strengthening the harmonics of $f_{D L}$. However, it is not expected that this numerical phenomenon significantly affects the circumferential position of the PSD maximum neither does it affect the qualitative trend of the PSD amplitude, which are discussed in the previous sections. Also, it should be noted that the Strouhal number corresponding to the PSD maximum is not the same harmonic of $S t_{D L}$ for all cases: for example, $S t_{\max } / S t_{D L}$ is about four in case $P / D=1.06$, whereas this ratio becomes five for case $P / D=1.03$. This possibly explains why the qualitative trend of $S t_{\max }$ is similar to Möller's correlation, whereas both datasets do not match quantitatively. For completeness, it should be stressed that this ratio does not yield a discrete value in two cases: it is equal to 6.7 for $P / D=1.02$ and 2.3 for $P / D=1.15$. However, the PSD contains several local maxima next to the absolute maximum described here. In case $P / D=1.02$, a local maximum occurs at $4 S t_{D L}$ and in case $P / D=1.15$, one occurs at $3 S t_{D L}$. This again indicates that, although the Strouhal number $S t_{\max }$ is likely to be influenced by the finite domain length, the qualitative trend found in Figure 12 is of physical nature.

${ }^{1}$ The phrase "close to" means: $S t_{\max }$ lies within ten percent of a multiple of $S t_{D L}$, although the margin was smaller than ten percent for most cases meeting this criterion. 


\subsubsection{Harmonic content of the PSD}

Figure 6 shows that the PSD does not exhibit a single maximum. In fact, up to a $P / D$-ratio of 1.085 , the function $\operatorname{PSD}(\theta, \operatorname{St})$ contains several maxima, all located at the same value of $\theta$, but at a different Strouhal number. The difference in Strouhal number between two adjacent maxima, $S t_{\text {shift }}$, is nearly constant, which leads to the assumption that the extrema indicate harmonics in the instability. Figure 13 shows the value of this frequency shift as a function of the $P / D$-ratio. It is noteworthy that multiple values per $P / D$-ratio were plotted, not just one value averaging the shifts between the different adjacent maxima (black dots). All black points at a certain $P / D$-ratio are close to each other, confirming the fact that the frequency shifts are either of physical or numerical nature and not due to noise. Additionally, there appears to be a local maximum at $P / D=1.03$, but looking at the remaining data points, the general conclusion is that the frequency shift increases with the $P / D$-ratio. The question is whether this harmonic content is a numerical or physical phenomenon. The only numerical parameter which could cause this PSD-characteristic, is the domain length. The Strouhal number $S t_{D L}$, which is defined in Equation (19), corresponds very well to the obtained values (red dots). This is a strong indication that the harmonic content observed in the PSD graph is a numerical phenomenon, related to the finite domain length and the predicted convection speed. In order to check the domain length's influence, another case is defined with the following geometrical parameters: $P / D=1.085$ and $L / D_{h}=22.5$. Table 4 contains the case characteristics and allows the comparison to the case where $L / D_{h}=15$, which was used previously (this is case $P / D=1.085$ in Table 3). According to Equation (19), the theoretical variable $S t_{D L}$ is equal to 0.043 . Using the procedure described in Section 5, it was verified that the convection speed $U_{c}$ did not yield a significantly different value compared to the case where $L / D=15$, such that Equation (20) is applicable. The PSD profile of the newly-defined case showed a nearly constant frequency shift between two local PSD maxima as well. This Strouhal number shift lies within a 5\% margin of $S t_{D L}$. This confirms the hypothesis that the domain length influences the harmonic shift in the PSD-profile. Consequently, this shift is likely to be a numerical phenomenon. Finally, it should be noted that $S t_{D L}$ and the frequency shift from the simulation do not match for $P / D=1.04$. There is no clear reason for this discrepancy.

Table 4: Non-dimensional characteristics of two cases computed to analyze the influence of the harmonic shift in the PSD. For all cases, $R e_{D_{h}}$ equals 10,000 and $P / D=1.085$.

\begin{tabular}{|c|c|c|c|c|}
\hline Case & $L / D_{h}$ & $\Delta t U / \Delta z$ & $N_{\text {eval }}$ & Cells \\
\hline$L / D_{h}=15$ & 15 & 0.268 & 48,000 & $4,800,000$ \\
$L / D_{h}=22.5$ & 22.5 & 0.268 & 54,000 & $7,200,000$ \\
\hline
\end{tabular}




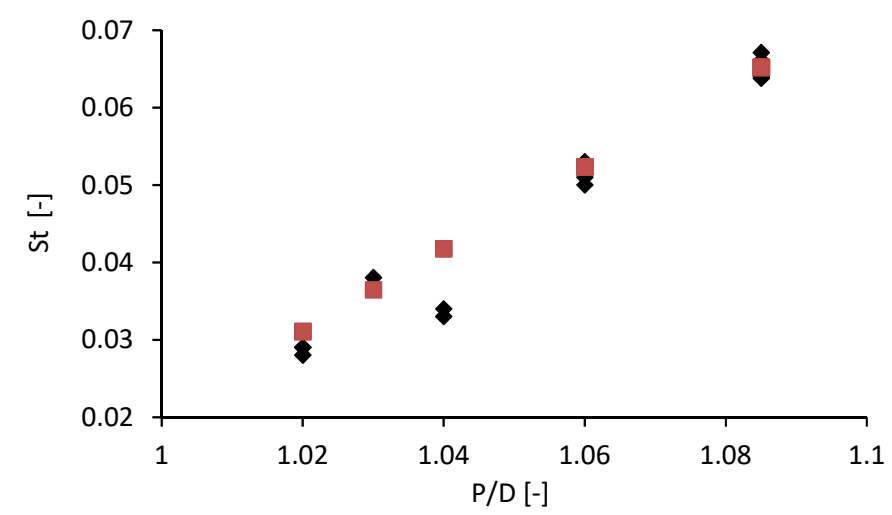

Figure 13: Variation of the Strouhal number shift between adjacent PSD maxima as a function of $P / D$. The black diamonds show the simulation results $\left(S t_{\text {shift }}\right)$. The characteristic Strouhal number $S t_{D L}$ is depicted by the red squares.

\section{Analysis of the CSD}

Some flow characteristics cannot be estimated with the PSD. This is due to the definition of the PSD: the two coordinates $\Delta \theta$ and $\Delta z$ are set to zero. This effectively means that each term composing the PSD contains information about only one location in space. However, in order to determine the convection speed, the pressure profiles at different locations on the cylinder wall need to be compared, as is the case in the more general definition of the CSD. The frequency- and space-dependent CSD therefore provides valuable information to further understand the axial flow through an array of cylinders. Because the main interest of this research is to determine the convection speed of the instability, only the axial coordinate is varied, while keeping the circumferential position fixed: $\operatorname{CSD}\left(\theta_{1}, \theta_{1}, \Delta z, S t\right)$.

The function $\operatorname{CSD}\left(\theta_{1}, \theta_{1}, \Delta z, S t\right)$ is periodic over $90^{\circ}$, as assumed in Equation (11).

The coherence is defined similar to Equation 12: $\gamma\left(\theta_{1}, \theta_{1}, \Delta z, S t\right)=\operatorname{CSD}\left(\theta_{1}, \theta_{1}, \Delta z, S t\right) / \operatorname{PSD}\left(\theta_{1}, S t\right)$.

Every term in the definition of the CSD thus consists of the product of the

Fourier variables of two points having the same circumferential coordinate, but

a different axial position.

\subsection{Characteristic shape}

Figure 14 shows the amplitude and phase angle of the function $\gamma\left(\theta_{1}, \theta_{1}, \Delta z, S t\right)$. The vertical axis shows the differential axial position $\Delta z$ and the horizontal axis shows the Strouhal number. The $P / D$-ratio is equal to 1.085 and the angle $\theta_{1}$ is set to zero. Firstly, the coherence amplitude becomes one and the phase angle becomes zero for $\Delta z$ equal to zero: this is due to the definition of the coherence. Secondly, both the amplitude and phase plots exhibit hyperbolically-shaped bands. Moreover, the bands in the amplitude and phase plot are correlated: the coherence amplitude is minimal when the phase angle is $180^{\circ}$ and the amplitude is maximal when the phase angle equals $0^{\circ}$. 


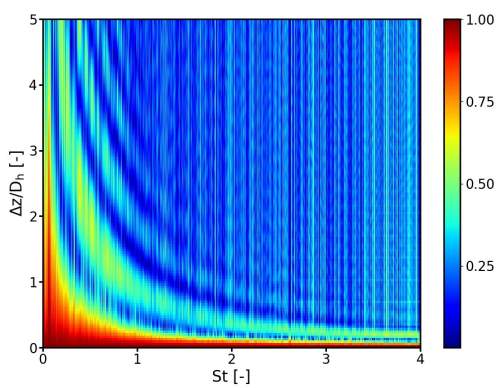

(a)

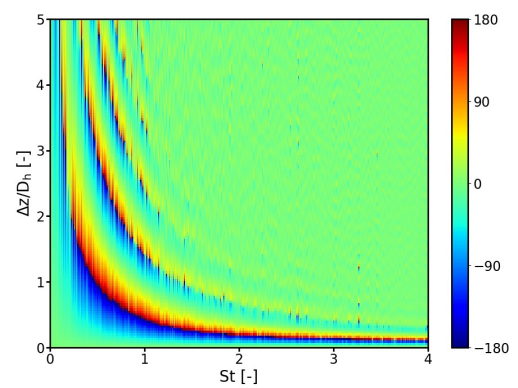

(b)

Figure 14: Coherence $\gamma(0,0, \Delta \mathrm{z}, \mathrm{St})$ in case $P / D=1.085$ with $\Delta z / D_{h}$ up to 5. (a) Amplitude (b) Phase

The occurrence of hyperbolic bands in the CSD profile has been observed before. De Ridder et al. (2016) performed Large-Eddy Simulations on an annular flow geometry with a Reynolds number equal to 127,000 and found a similar profile for the CSD. The explanation for the CSD bands presented in that paper is based solely on the transport of a perfect, non-viscous vortex structure through the array. Although the bands in the phase plot can be explained by the presence of moving waves, it does not clarify the amplitude behaviour. If two points on the cylinder surface, which are positioned $\Delta z$ apart, see a similar pressure profile with a time delay $\frac{\Delta z}{U_{c}}$ due to the finite transport speed of the instability, the amplitude does not change as a function of $\Delta z$. If the pressure profile in the first point is symbolized by $x(t)$ and in the second point by $y(t)-t$ being the continuous time parameter- the Fourier variables in the CSD would be as follows:

$$
\begin{gathered}
y(t)=x\left(t-\frac{\Delta z}{U_{c}}\right) \leftrightarrow Y(k)=X(k) \exp \left(-j \frac{2 \pi k}{T} \frac{\Delta z}{U_{c}}\right) \\
X(k)^{*} Y(k)=X(k)^{*} X(k) \exp \left(-j \frac{2 \pi k}{T} \frac{\Delta z}{U_{c}}\right)=|X(k)|^{2} \exp \left(-j \frac{2 \pi k}{T} \frac{\Delta z}{U_{c}}\right)
\end{gathered}
$$

where $X(k)$ is the DFT of signal $x(t), X^{*}$ is the complex conjugate of $X$ and $\cdot \mid$ is the amplitude of a complex variable. According to the CSD definition, this product is subsequently used in a summation over the axial coordinate. As it is assumed that the same pressure profile is seen over the entire cylinder - with a time delay dependent on the relative position of the points on the wall - the theoretical $X(k)$ is not dependent on the axial position. The conclusion is that the amplitude of the coherence would be independent of the parameter $\Delta z$, which is clearly not the case. In other words, the coherence bands cannot be explained completely with a moving wave hypothesis. Additionally, it should be noted that the global coherence profile is not hyperbolic: due to the periodic boundary conditions, the CSD profile is relatively symmetric around half of the cylinder length $\left(\Delta z / D_{h}=7.5\right.$ in this particular case). This is shown in 
Figure 15, in which the parameter $\Delta z / D_{h}$ is extended to 15 , corresponding to the entire domain length.

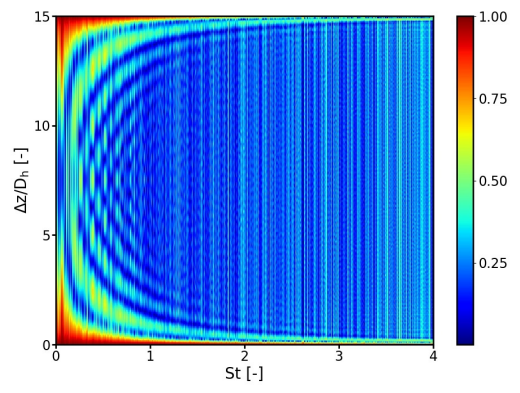

(a)

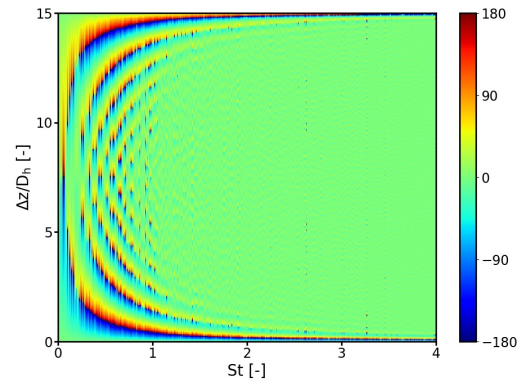

(b)

Figure 15: Coherence $\gamma(0,0, \Delta \mathrm{z}, \mathrm{St})$ in case $P / D=1.085$ and $L / D_{h}=15$ with $\Delta z / D_{h}$ up to 15. (a) Amplitude (b) Phase

The coherence profile in Figure 15 possibly indicates the presence of standing waves in the flow domain. Standing waves would influence the coherence amplitude, but the phase behaviour cannot be the result of a standing wave: only $0^{\circ}$ and $180^{\circ}$ phase change can occur in a standing wave. Consequently, it is hypothesized that the presence of both standing and travelling waves in the flow domain lead to the observed CSD. This is verified by automatically generating pressure data of known wave forms and subsequently calculating the coherence function. This theoretical pressure profile is applied to an array of points along a straight line, which is similar to a set of points with the same circumferential coordinate, but located at different axial positions. The presumed Reynolds number is 10,000 . The ratios $P / D$ and $L / D_{h}$ are chosen equal to 1.085 and 15 , respectively. The ratio $U_{c} / U$ is set to 1.02 , which is the mean value obtained for $P / D=1.085$ in paragraph 5.3. The spatial resolution is kept the same as in the original case $P / D=1.085$, i.e. 400 points distributed over a total length of $15 D_{h}$. The theoretical pressure profile is dependent on one spatial parameter $z$ and on the time instant $t$. It is defined as:

$$
\begin{aligned}
p(z, t) & =\sum_{m=1}^{60} \sin \left(2 \pi m \frac{U_{c} t}{L}\right) \sin \left(2 \pi m \frac{z}{L / 2}\right) \\
& +\sum_{m=1}^{60} \sin \left(2 \pi m \frac{U_{c}}{L}\left[t-\frac{z}{U_{c}}\right]\right)
\end{aligned}
$$

The standing wave time frequencies are chosen equal to a multiple of $U_{c} / L$ and the spatial wavelengths are set to a multiple of $L / 2$. For the travelling waves, the frequency is a multiple of $U_{c} / L$. As such, the periodic boundary conditions are reproduced in the theoretical pressure profile. The resulting coherence function (for which no circumferential coordinate is determined) is shown in Figure 
16. These graphs strongly resemble Figures 14 and 15, which is an indication that the proposed hypothesis is correct. There are two discrepancies between the theoretical coherence profile in Figures 15 and 16. The first one is at $S t=0$. As shown in Equation 23, the very low frequencies are not excited and, as such, the coherence amplitude is minimal whereas it is maximal in the coherence obtained from the simulations. Secondly, the coherence obtained from LES fades quickly for an increasing value of $\Delta z$. Due to other perturbations, i.e. turbulence or the generation of new Kelvin-Helmholtz instabilities, the pressure profile is distorted during the transport in the axial direction. Consequently, the coherence amplitude maximum value decreases for increasing $\Delta z$. Also, the hyperbolic bands disappear for high Strouhal numbers. High frequencies correspond to smaller vortices, which are more sensitive to dissipation by turbulence. This leads to a reduced pressure correlation and a corresponding phase shift, which becomes zero on average.

In the previous paragraph, it has been established that the coherence function behaviour is due to both a standing and a travelling wave phenomenon. The latter is intuitively understood, as it is related to the transport of an instability through the flow domain. This is why the convection speed $U_{c}$ was used in Equation (23). By contrast, the standing waves are counter-intuitive. This effect is not related to the finite domain length $L$, even though $L$ appears in Equation (23). In order to confirm this, the coherence is calculated for case $L / D_{h}=22.5$ described in Table 4 . The amplitude and phase of this coherence function are shown in Figure 17. The bands in Figures $17 \mathrm{~b}$ and $17 \mathrm{~d}$ are located on the same position as for the smaller domain length $L / D_{h}=15$. The difference between both cases is that the central region in Figures $17 \mathrm{a}$ and $17 \mathrm{c}$ is elongated, because the bands around $\Delta z / D_{h}=0$ are similar in both cases, independent of the domain length.

The explanation for the standing wave phenomenon is not related to the domain length, but it is due to another numerical aspect: the definition of the pressure reference point. In incompressible flow, the Navier-Stokes equations only define the pressure gradient. Consequently, if an appropriate pressure field $p^{*}$ is found, any pressure profile $p=p^{*}+C$ (where $\mathrm{C}$ is a constant value) is also a valid solution. In order to overcome this ambiguity, the OpenFOAM solver defines a pressure reference point at which the pressure is set to zero gauge pressure by definition. In all time steps, the pressure in this point is constant and the flow field is calculated accordingly. The periodic flow instability which is transported through the domain consists of a number of frequency components. If the time frequency of one of these components is denoted with $f$, the corresponding spatial wavelength is: $\lambda=U_{c} / f$. Therefore, it is expected that the points located at an axial distance equal to (a multiple of) $\lambda$ from the pressure reference point also experience (nearly) zero pressure. The location of these pressure nodes is thus related to the transport of the flow instability and is dependent on the frequency (or, equivalently, the Strouhal number) as well as the convection speed. This explains why the convection speed occurs in the expression for the standing wave and also why the pressure nodes are not located on the same axial position for all Strouhal numbers. In order to verify 


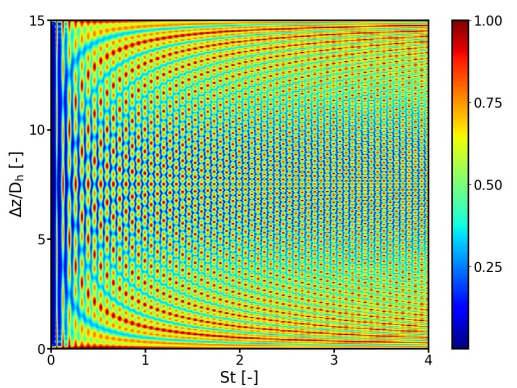

(a)

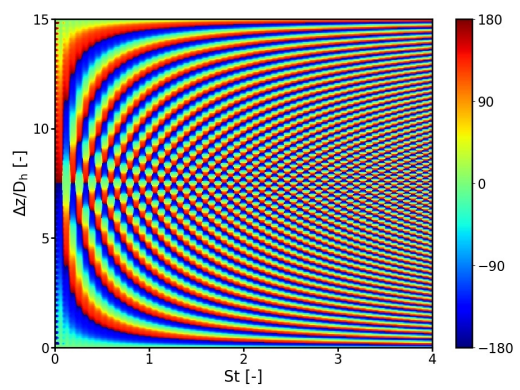

(c)

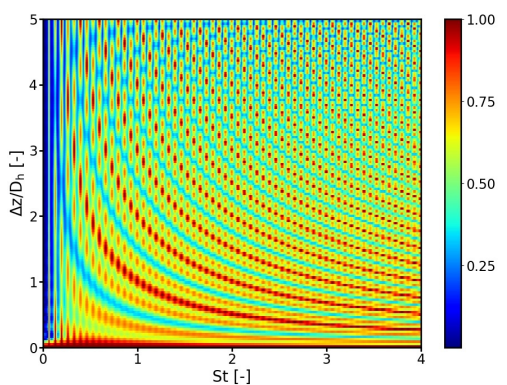

(b)

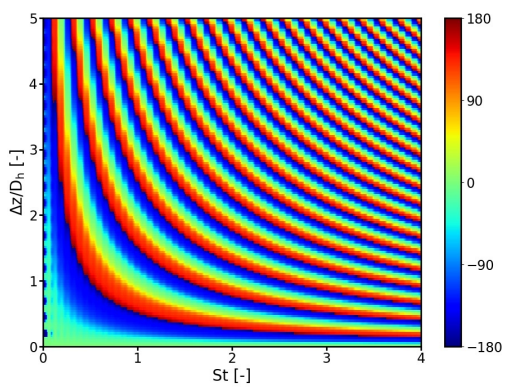

(d)

Figure 16: Coherence $\gamma(\Delta z, S t)$ of the theoretical pressure profile defined in Equation (23). (a) Amplitude, with $\Delta z / D_{h}$ up to 15 (b) Amplitude, with $\Delta z / D_{h}$ up to 5 (c) Phase, with $\Delta z / D_{h}$ up to 15 (d) Phase, with $\Delta z / D_{h}$ up to 5 


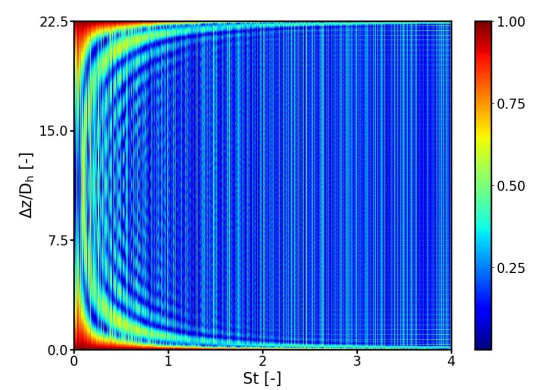

(a)

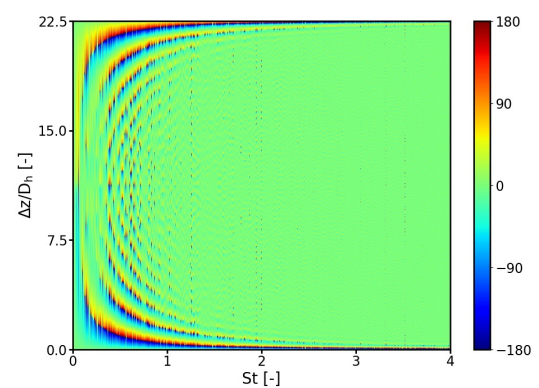

(c)

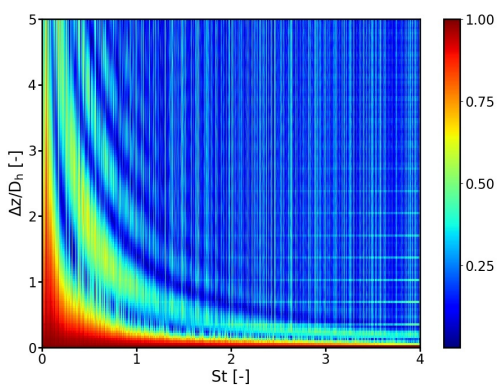

(b)

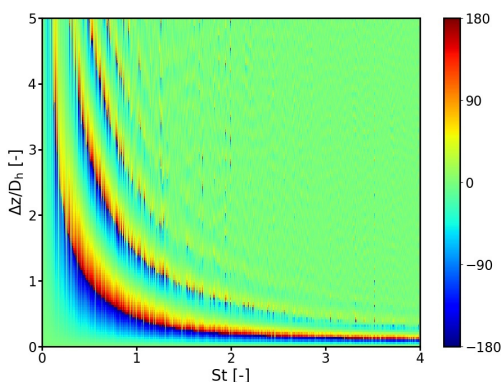

(d)

Figure 17: Coherence $\gamma(0,0, \Delta \mathrm{z}, \mathrm{St})$ in case $P / D=1.085$ and $L / D_{h}=22.5$. (a) Amplitude, with $\Delta z / D_{h}$ up to 22.5 (b) Amplitude, with $\Delta z / D_{h}$ up to 5 (c) Phase, with $\Delta z / D_{h}$ up to 22.5 (d) Phase, with $\Delta z / D_{h}$ up to 5 
this reasoning, the radial force $F_{r 0}$ in the direction of $\theta=0^{\circ}$ is calculated with a discrete approximation of the pressure integral:

$$
F_{r 0}=\sum_{m=0}^{N_{\theta}-1} p \cos \left(m \Delta \theta_{c e l l}\right) A_{\text {cell }}
$$

where $N_{\theta}$ represents the number of divisions in the circumferential direction and $\Delta \theta_{\text {cell }}$ is the circumferential angle taken by a single cell. Finally, $A_{\text {cell }}$ is the size of the cell face on the wall. By performing the CSD operation on the force $F_{r 0}$, the influence of the pressure reference point should be removed. The definition of the CSD in Equations (10) and (11) is directly applicable if the pressure is replaced by the radial force. In that case, the CSD is no longer dependent on the circumferential position as was the case for the pressure profile. The coherence of the radial force is denoted with $\gamma_{F}$. The coherence amplitude and phase in the case of $P / D=1.085$ and $L / D_{h}=22.5$ are shown in Figure 18. The bands in the phase plot are the same as in Figure 17, but the bands in the coherence amplitude are almost invisible. The disappearance of the bands seems to support the hypothesis that the standing wave phenomenon is related to the pressure reference point. The decay of the coherence amplitude for higher $\Delta z$ and $S t$ has already been discussed.

Finally, the phase angle shift of the coherence is solely related to the finite velocity at which the vortex structure is transported, i.e. the travelling waves in the flow domain. The axial distance over which the coherence undergoes a $360^{\circ}$ phase shift is therefore equal to the distance travelled by the KelvinHelmholtz instability during one oscillation period $T=1 / f=D_{h} /(S t U)$. In other words, the axial length between two adjacent bands in the coherence phase graph, further denoted by $\Delta z_{\text {band }}$, is a measure of the convection speed $U_{c}$, which is the speed at which the coherent structures are transported axially through the domain.

\subsection{Influence of the circumferential coordinate}

In this section, the influence of the parameter $\theta_{1}$ on $\gamma\left(\theta_{1}, \theta_{1}, \Delta z, S t\right)$ is discussed. Curling and Païdoussis (1992) states that when the angle $\theta_{1}$ is chosen closer to the subchannel region, the convection speed increases and the hyperbolic bands in the CSD-profile come closer together. In order to verify this, the phase angle of the coherence function $\gamma\left(\theta_{1}, \theta_{1}, 3 D_{h}, S t\right)$ from the LES calculation are superimposed on Curling's correlation in Figure 19. The dataset from the numerical analysis does not show a noticeable difference between the gap and subchannel region. Additionally, Guellouz and Tavoularis (2000) postulated Equation (25) for the convection of the flow instability, which indirectly means that the convection speed is independent of $\theta_{1}$ (at least for small $\theta_{1}$, close to the gap region):

$$
\frac{U_{c}}{U}=1.04\left(1-\exp \left(-10.9 \frac{P}{D}+10.6\right)\right) .
$$




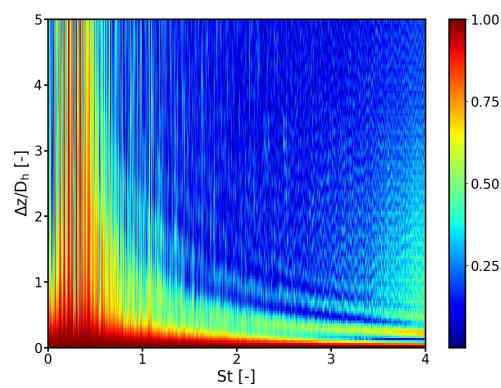

(a)

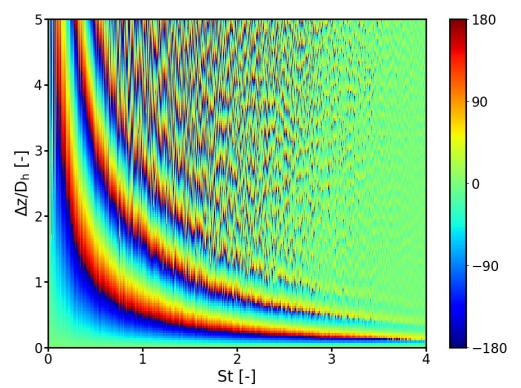

(b)

Figure 18: Coherence $\gamma_{\mathrm{F}}(\Delta \mathrm{z}, \mathrm{St})$ of the radial force in case $P / D=1.085$ and $L / D_{h}=22.5$. (a) Amplitude (b) Phase

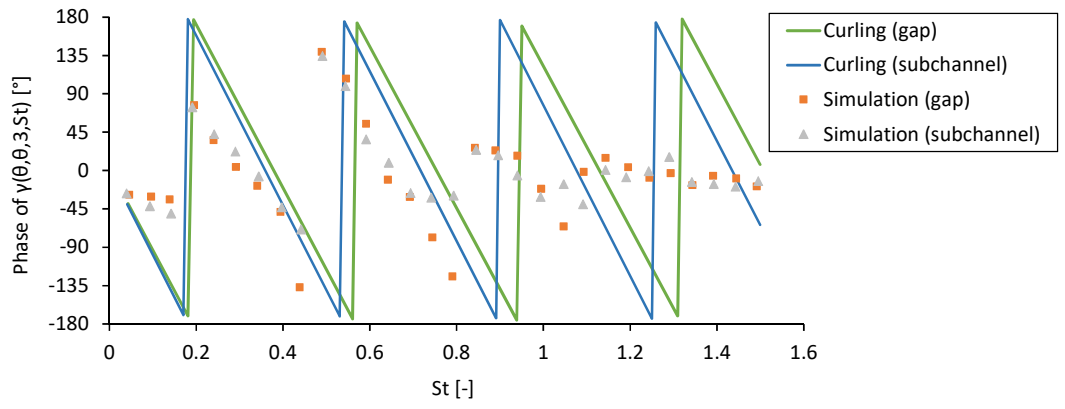

Figure 19: Phase of the coherence $\gamma\left(\theta_{1}, \theta_{1}, 3 D_{h}, S t\right)$ in the gap $\left(\theta_{1}=0^{\circ}\right)$ and subchannel region $\left(\theta_{1}=45^{\circ}\right)$ for case $\mathrm{X} 50$ in Table $1(R e=14,000, P / D=1.085)$. The dots are the present simulation results, the lines show Curling's correlation (Curling and Païdoussis (1992)). 
Consequently, there is some uncertainty about the influence of the angle $\theta_{1}$. It is noted that Guellouz's correlation only considers the coherent structures, whereas Curling's experiments also show a significant turbulence level. Still, as the turbulence level in the presented simulations is low, Guellouz's correlation is assumed to be a good description of the convection speed.

The following analysis is proposed: the CSD is calculated for $\theta_{1}=0^{\circ}$ and $\theta_{1}=45^{\circ}$, respectively. For each of these plots, the axial distance between two adjacent bands was determined at Strouhal numbers equal to $0.3,0.5$ and 1 , respectively. The results are shown in Figure 20. For now, only the red datapoints are compared to the blue ones. The red datapoints result from the coherence at $\theta_{1}=0^{\circ}$ especially for a Strouhal number equal to 0.3 and 0.5 , the distance between adjacent bands $\Delta z$ seems to be equal. For a Strouhal number of 1 , the variation is apparently somewhat higher, due to the decay of the coherence phase bands for larger Strouhal numbers, but still there is no clear distinction between both datasets. Increasing the Strouhal number beyond 1 is impossible for most cases, as the spatial period cannot be discerned with sufficient accuracy due to the fading of the CSD bands.

Although the noise on the CSD could hide a minor influence of the angle $\theta_{1}$, the simulations do not show any dependence of the CSD on the parameter $\theta_{1}$. This leads to the conclusion that the coherent structures are transported as a whole; the vortices outside of the gap region and the cross-flow inside the gap region are not moving relative to each other. No experimental (Meyer (2010); Curling and Païdoussis (1992)) evidence has indicated otherwise so far.

\subsection{Influence of the cylinder spacing}

The qualitative image of the coherence function $\gamma\left(\theta_{1}, \theta_{1}, \Delta z, S t\right)$, as shown in Figure 14, does not change when altering the $P / D$-ratio, but the width between the CSD bands is dependent on the cylinder spacing. The hypothesis is that the flow speed difference between the gap and subchannel region decreases for increasing cylinder spacing. It is logical that this change will affect the speed at which the coherent structures are transported in the axial direction, the socalled convection speed $U_{c}$. It was noted in Section 5.1 that the convection speed is determined indirectly from the coherence graph. To do so, a Strouhal number is chosen beforehand and the corresponding axial separation $\Delta z_{\text {band }}$ corresponding to a $360^{\circ}$ phase shift is measured. This band width, $\Delta z_{\text {band }}$, is the distance covered by the pressure fluctuation corresponding to the predetermined Strouhal number during one oscillation period. As such, the convection speed equals

$$
U_{c}=\Delta z_{\text {band }} f .
$$

Obviously, the resulting vortex street is the superposition of all individual instabilities, each at a different frequency $f$ (or corresponding Strouhal number $S t)$. $U_{c}$ should be determined at different frequencies, which is done for three different Strouhal numbers: $0.3,0.5$ and 1. For the graphs corresponding to the Strouhal numbers 0.5 and 1 in Figure 20, it is observed that $\Delta z$ is halved when the frequency is doubled, therefore indicating that $U_{c}$ is a constant value for a 


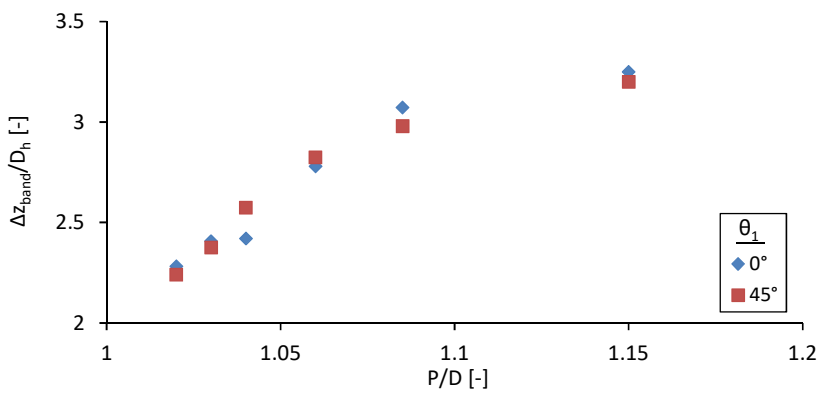

(a)

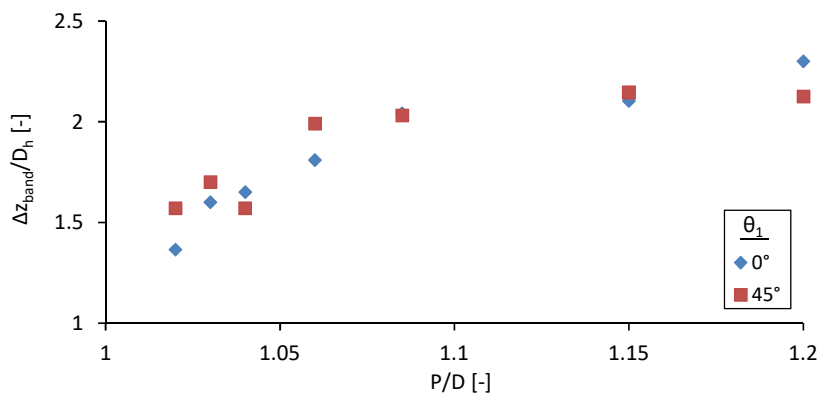

(b)

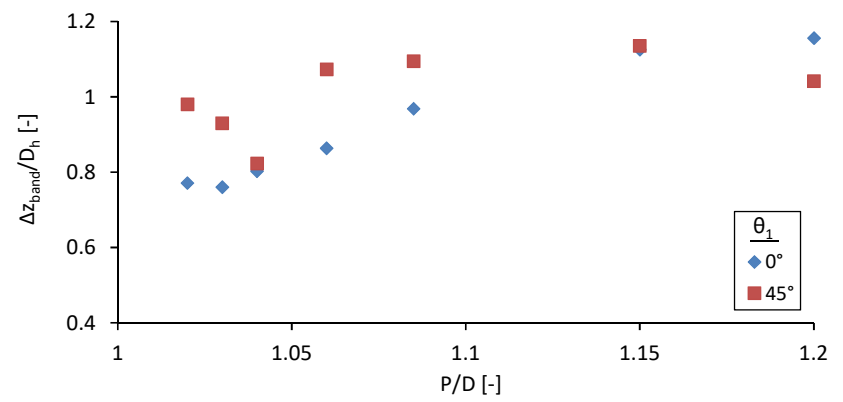

(c)

Figure 20: Variation of the axial distance $\Delta z_{\text {band }}$ between adjacent CSD bands as a function of the $P / D$-ratio for $\theta_{1}=0^{\circ}$ and $45^{\circ}$. (a) $\mathrm{St}=0.3$. The value of $\Delta z_{\text {band }}$ for $P / D=1.2$ could not be determined accurately due to noise and has therefore been omitted. (b) St $=0.5$. (c) St $=1.0$. 
certain geometry. However, this conclusion is not entirely valid: from Figure 20 and Equation (26), it is clear that the convection speed is significantly lower for $S t=0.3$ than for the other two Strouhal numbers. In other words, the convection speed $U_{c}$ is not solely dependent on the $P / D$-ratio, but decreases somewhat for low-frequency components. This is contradictory to the assumption made by Guellouz and Tavoularis (2000). Although the LES results show some dependence of the convection speed on the Strouhal number, the discrepancy between lower and higher Strouhal numbers is neglected in the following analysis. In order to determine the dependence on the $P / D$-ratio, the convection speed is calculated with Equation (26) for all datapoints presented in Figure 20. As there is no dependence on $\theta_{1}$, the values for $\Delta z_{\text {band }}$ are calculated with the average of the results for $\theta_{1}$ equal to $0^{\circ}$ and $45^{\circ}$. The resulting convection speed $U_{c}$ is shown as a function of the $P / D$-ratio, in Figure 21. Strouhal numbers equal to 0.5 and 1 respectively, yield similar values for the convection speed (blue dots). The only exception is in case $P / D=1.02$, where the value for $U_{c}$ at a Strouhal number equal to 1 was so large compared to other values, that it was deemed reasonable to remove this outlier from the graph. However, a Strouhal number equal to 0.3 gives values for the convection speed which are roughly 10 percent lower than for the higher Strouhal numbers. Additionally, notice the monotonous increase of $U_{c} / U$ for increasing $P / D$-ratio. As the space between the cylinders becomes larger, the instability is spread over a larger distance, hereby increasing $\Delta z_{\text {band }}$.

The correlation defined by Guellouz and Tavoularis (2000) (Equation (25)) is shown as a red line in Figure 21. The simulation data exhibit a similar behaviour as this correlation, although at a consistently higher value. Consequently, an improved correlation (shown as a green curve in Figure 21) is proposed by altering the coefficients in Guellouz's correlation:

$$
\frac{U_{c}}{U}=1.11\left(1-\exp \left(-17 \frac{P}{D}+16.3\right)\right),
$$

As can be seen from Figure 21, this ratio is larger than one for $P / D$-ratios above 1.1. This result is unexpected, because one would assume that the convection speed would become equal to $U$ when the cylinder spacing is large: the distinction between gap and subchannel disappears and the entire flow experiences approximately the same flow speed (except for the region close to the cylinder wall). However, one should take into account that the convection speed is only relevant up to a certain $P / D$-ratio. Indeed, if the driving force behind the instability - the flow speed difference between the gap and subchannel region - decays, the coherent structures will weaken and eventually disappear altogether, rendering the term "convection speed" moot. Consequently, the correlation should only be evaluated for relatively small $P / D$-ratios. Considering the discussion about the PSD in previous chapter, a limit value of $P / D=1.2$ is a logical choice. $U_{c} / U$ is larger than one for the range $P / D=1.1-1.2$. As $U$ is not the maximal flow speed but the mean flow speed, this is possible. The convection speed $U_{c}$ is expected to be somewhere between the gap and subchannel region speeds, depending on the exact location of the vortices. If 
the bulk of the vortex street is located more towards the subchannel region, the convection speed is likely to be higher. In other words, $U_{c} / U>1$ indicates that the vortices leading to the largest pressure fluctuation on the cylinder wall are located more towards the subchannel region.

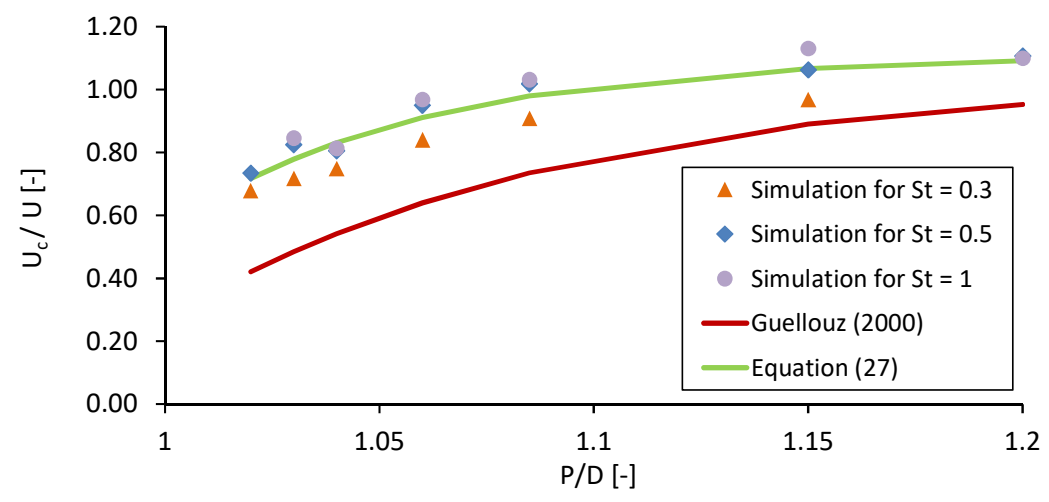

Figure 21: Ratio of the convection speed to the flow speed $\frac{\mathrm{U}_{\mathrm{c}}}{\mathrm{U}}$ as a function of the $P / D$-ratio.

\section{Conclusion}

Large-Eddy Simulations are performed on a single-cylinder approximation of an infinite array of cylinders subjected to external axial flow. The aim of this paper is to comprehend the behaviour of the coherent vortex structures occurring in this flow. These coherent structures exist due to the axial flow speed difference between the gap and subchannel regions in the geometry, resulting in a Kelvin-Helmholtz instabilty. The pressure profile on the cylinder wall obtained from the CFD calculations is used in a spectral analysis.

Regarding the numerical aspects, this paper shows that the influence of the single-cylinder domain boundaries on the flow is not negligible. The finite domain length and the periodic in- and outlet conditions force a discrete number of eddies into the flow domain, therefore altering the frequency content of the pressure profile on the cylinder wall. These boundary conditions cannot be altered without neglecting the assumption of an infinite array of infinitely long cylinders. However, the computational effort is severely reduced compared to the analysis of a flow domain containing multiple cylinders. Moreover, the present analysis shows that the single-cylinder approximation still provides useful information to enhance the physical understanding of the flow instability.

The strength of the flow instability is influenced significantly by the cylinder spacing, quantified through the pitch-over-diameter $(P / D)$ ratio. Up to a $P / D$-ratio equal to 1.03 , the instability becomes stronger for increasing cylinder spacing due to the reduced friction in the gap region. For higher $P / D$-ratios, 
the instability weakens exponentially due to the decreasing difference between the gap and subchannel regions. The circumferential position on the cylinder wall at which the highest pressure is observed, shifts from the gap to the subchannel region for increasing cylinder spacing. As the bulk of the large-scale vortices are shifted towards the subchannel region, the convection speed also increases. An improved correlation for the convection speed as a function of the $P / D$-ratio is proposed. The influence of the Strouhal number on the convection speed is small for relatively large Strouhal numbers and is therefore neglected. The circumferential position at which the convection speed is determined, does not influence the result.

\section{Acknowledgments}

This work was performed in the framework of the Horizon 2020 SESAME Project. It has received funding from the European Commission Euratom Research and Training Programme on Nuclear Energy under grant agreement No 654935 (SESAME). The authors gratefully acknowledge the funding by the Research Foundation-Flanders (FWO), through the Ph.D. fellowship of Laurent De Moerloose. The computational resources (Stevin Supercomputer Infrastructure) and services used in this work were provided by the VSC (Flemish Supercomputer Center), funded by Ghent University, the Hercules Foundation and the Flemish Government department EWI.

\section{References}

Abbasian, F., Yu, S., Cao, J., 2010. Large eddy simulation of turbulent flow along an array of rods. Journal of Fluids Engineering 132 (021105), 1-11.

Au-Yang, M., 2001. Flow-Induced Vibration of Power and Process Plant Components. Professional Engineering Publishing.

Curling, L., Païdoussis, M., 1992. Measurements and characterization of wallpressure fluctuations on cylinders in a bundle in turbulent axial flow. Academic Press 157 (3), 435-449.

De Ridder, J., Degroote, J., Van Tichelen, K., Schuurmans, P., Vierendeels, J., 2016. Predicting turbulence-induced vibration in axial annular flow by means of large-eddy simulations. Journal of Fluids and Structures 61, 115-131.

Guellouz, M., Tavoularis, S., 2000. The structure of turbulent flows in a rectangular channel with a cylindrical rod. Experimental Thermal and Fluid Science 23, 59-91.

Kakaç, S., Liu, H., Pramuanjaroenkij, A., 2012. Heat Exchangers: Selection, Rating and Thermal Design. CRC Press. 
Lexmond, A., Mudde, M., Van der Haagen, M., 2005. Visualization of the vortex street and characterization of the cross flow in the gap between two subchannels. Proceeding of 11th Nureth conference, Avignon, paper: 122.

Meneveau, C., Lund, T., Cabot, W., 1996. A lagrangian dynamic subgrid-scale model of turbulence. Journal of Fluid Mechanics 319, 353-385.

Merzari, E., Fischer, P., Yuan, H., Van Tichelen, K., Keijers, S., De Ridder, J., Degroote, J., Vierendeels, J., Doolaard, H., Gopala, V., Roelofs, F., 2016. Benchmark exercise for fluid flow simulations in a liquid metal fast reactor fuel assembly. Nuclear Engineering and Design 298, 218-228.

Merzari, E., Ninokata, H., Baglietto, E., 2008. Numerical simulation of flows in tight-lattice fuel bundles. Nuclear Engineering and Design 238, 1703-1719.

Meyer, L., 2010. From discovery to recognition of periodic large scale vortices in rod bundles as source of natural mixing between subchannelsa review. Nuclear Engineering and Design 240, 1575 - 1588.

Modarres-Sadeghi, Y., Païdoussis, M., Semler, C., 2007. The nonlinear behaviour of a slender flexible cylinder pinned or clamped at both ends and subjected to axial flow. Computers and Structures 85, $1121-1133$.

Möller, S., 1991. On phenomena of turbulent flow through rod bundles. Experimental Thermal and Fluid Science 4 (1), 25-35.

Nikuradse, J., 1930. Untersuchungen über turbulente Strömungen in nicht kreisförmigen Rohren. Ingenieur Archiv 1, 306-332.

Païdoussis, M., 1982. A review of flow-induced vibrations in reactors and reactor components. Nuclear Engineering and Design 74, 31-60.

Païdoussis, M., 1998. Fluid-structure interactions: Slender Structures and Axial Flow: Volumes 1 and 2. Academic Press.

Païdoussis, M., Botez, R. M., 1993. Chaotic dynamics of articulated cylinders in confined axial flow. Journal of Fluids and Structures 7, 719-750.

Pettigrew, M., Taylor, C., 2003. Vibration analysis of shell-and-tube heat exchangers: an overview-part 1: flow, damping, fluidelastic instability. Journal of Fluids and Structures 18 (5), 469-483.

Piomelli, U., 2012. Large eddy simulation and related techniques: Theory and applications. Lecture series at the von Karman Institute for Fluid Dynamics.

Yan, B., Yu, L., 2011. URANS simulation of the turbulent flow in a tight lattice: Effect of the pitch to diameter ratio. Progress in Nuclear Energy 53, 428-437. 